\title{
F-Actin Bending Facilitates Net Actomyosin Contraction By Inhibiting Expansion With Plus-End-Located Myosin Motors
}

\author{
Alexander K. Y. Tam*1, Alex Mogilner ${ }^{2}$, and Dietmar B. Oelz ${ }^{1}$ \\ ${ }^{1}$ School of Mathematics and Physics, The University of Queensland, St Lucia, Queensland 4072, \\ Australia. \\ ${ }^{2}$ Courant Institute of Mathematical Sciences, New York University, New York, NY, USA
}

August 20, 2021

\begin{abstract}
We investigate whether a microscopic system of two semi-flexible actin filaments with an attached myosin motor can facilitate contraction. Based on energy minimisation, we derive and analyse a partial differential equation model for a two-filament-motor structure embedded within a dense, two-dimensional network. Our method enables calculation of the plane stress tensor, providing a measure for contractility. After deriving the model, we use a combination of asymptotic analysis and numerical solutions to show how F-actin bending facilitates net contraction as a myosin motor traverses two symmetric filaments. Myosin motors close to the minus-ends facilitate contraction, whereas motors close to the plus-ends facilitate expansion. The leading-order solution for rigid filaments exhibits polarity-reversal symmetry, such that the contractile and expansive components balance to zero. Surprisingly, after introducing bending the first-order correction to stress indicates expansion. However, numerical solutions show that filament bending induces a geometric asymmetry that brings the filaments closer to parallel as a myosin motor approaches their plus-ends. This decreases the effective spring force opposing motion of the motor, enabling it to move faster close to filament plus-ends. This reduces the contribution of expansive stress, giving rise to net contraction. Further numerical solutions confirm that this applies beyond the small bending regime considered in the asymptotic analysis. Our findings confirm that filament bending gives rise to microscopic-scale actomyosin contraction, and provides a possible explanation for network-scale contraction.
\end{abstract}

Keywords: actomyosin, curve-straightening flow, energy functional, gradient flow, stress tensor, asymptotic analysis

\section{Introduction}

2 The mechanics of actin filament and myosin motor proteins in the cell cortex underpins 3 movement (Yamada and Sixt 2019) and division (Pollard 2010) of biological cells. Early

\footnotetext{
${ }^{*}$ Corresponding author: alex.tam@uq.edu.au
} 
breakthroughs in understanding actomyosin dynamics occurred in the context of muscle cells (Gautel 2011). There, actin and myosin form sarcomere structures, in which filaments are aligned in parallel with minus-ends in the centre and plus-ends pointing outwards. Relative motion of myosin motors towards filament plus-ends subsequently generates contraction by pulling filaments inwards. This mechanism is known as sliding filament theory (Huxley 2004). However, actomyosin networks in the cell cortex are disordered, with filaments distributed at random. Experiments (Murrell, Oakes, et al. 2015; Pollard and O'Shaughnessy 2019) and simulations (Tam, Mogilner, and Oelz 2021; Ennomani et al. 2016) have shown that disordered actomyosin networks also contract (Chalut and Paluch 2016). Sliding filament theory predicts that random networks would produce expansion or contraction with equal probability, and thus cannot explain this contraction. Consequently, the origin of contraction in disordered networks remains an active field of research. A pertinent question is whether asymmetry on the microscopic scale (Lenz 2014; Komianos and Papoian 2018) can explain network-scale contraction, or whether long-range effects amplify contractile stress (Ronceray, Broedersz, and Lenz 2016). A microscopic asymmetry would require additional mechanics to those predicted by sliding filament theory.

Filament bending flexibility is commonly-hypothesised as a source of asymmetry that might explain contraction of disordered actomyosin networks (Murrell and Gardel 2012; De La Cruz and Gardel 2015; du Roure et al. 2019; Head, Levine, and MacKintosh 2003; Tam, Mogilner, and Oelz 2021). Actin filaments are semi-flexible (Stachowiak et al. 2014; Belmonte, Leptin, and Nédélec 2017), such that they undergo small but significant bending (Broedersz and Mackintosh 2014; Murrell and Gardel 2012). This is not relevant in sarcomeres with parallel arrays of filaments, but is for two and three-dimensional networks with random filament orientations. Many previous experimental and theoretical studies invoke a filament buckling mechanism, whereby filaments can sustain longitudinal tension, but buckle under longitudinal compression (Bidone et al. 2017; Belmonte, Leptin, and Nédélec 2017; Cheffings, Burroughs, and Balasubramanian 2016; du Roure et al. 2019; Freedman, Banerjee, et al. 2017; Freedman, Hocky, et al. 2018; Lenz 2020; Murrell and Gardel 2012; Ronceray, Broedersz, and Lenz 2016; Soares e Silva et al. 2011; Yu et al. 2018). This has been shown to generate network-scale bias to contraction over expansion (Belmonte, Leptin, and Nédélec 2017). Other studies have considered a related filament bending mechanism (Lenz 2014; Tam, Mogilner, and Oelz 2021; Head, Levine, and MacKintosh 2003; Popov, Komianos, and Papoian 2016; Kim 2015; Letort et al. 2015) as a source of force asymmetry. This involves applying forces that pluck filaments transversely, as opposed to the longitudinal forces involved with buckling. Lenz (2014) showed that filament bending produces forces that exceed those involved with longitudinal buckling, and Tam, Mogilner, and Oelz (2021) showed that this mechanism facilitates network-scale contraction.

An interesting question is whether the force asymmetry provided by bending or buckling 
applies at the microscopic scale. De La Cruz and Gardel (2015) state that compression, stretching, and bending forces are relevant on the small scale. In contrast, Ronceray, Broedersz, and Lenz (2016) state that buckling facilitates network-scale contraction by enabling transmission of contractile force, and inhibiting expansive. This phenomenon is highly nonlinear, and does not require microscopic asymmetry. One approach to understand microscopic filament dynamics is to model a single filament as a worm-like chain (Broedersz and Mackintosh 2014; Lenz et al. 2012). Broedersz and Mackintosh (2014) used this approach to identify an asymmetry under extension and compression. Other authors have considered structures consisting of two-filaments and an attached motor (Lenz 2014; Belmonte, Leptin, and Nédélec 2017; Hiraiwa and Salbreux 2016; Komianos and Papoian 2018). Hiraiwa and Salbreux (2016) and Komianos and Papoian (2018) considered the effect of motors and cross-linkers on forces generated by rigid filament pairs. However, Lenz (2014) reported that disordered networks of rigid filaments with polarity-reversal symmetry (i.e. any configuration of filaments is equally likely as the same configuration with minus and plus-ends reversed) generate zero net contraction. Belmonte, Leptin, and Nédélec (2017) developed a statistical method to predict network-scale contraction by summing the contributions from two-filament structures, assuming filaments buckle under compression. An important advance was provided by Lenz (2014), who compared symmetry-breaking mechanisms for two filaments, and found filament bending gives rise to contraction on the microscopic scale. They subsequently showed bending to be the dominant mechanism of contraction for experimentally-feasible parameters. Our objective is to investigate the microscopic origin of bending-induced contraction in detail.

Semi-flexible filament evolution is an example of a curve-straightening flow. Mathematically, this refers to deformation of curves in $\mathbb{R}^{2}$ by decreasing their total squared curvature. Curve-straightening is relevant to actomyosin, because filaments evolve to minimise their bending energy, which is proportional to total curvature. Curve-straightening problems have been investigated extensively since the 1980s, mostly in the context of single curves (Langer and Singer 1984; Langer and Singer 1987; Linnér 1989; Linnér 2003). Wen (1993) and Wen (1995) then used the indicatrix representation and $L^{2}$-gradient flow of the squared curvature functional to derive a fourth-order, semilinear parabolic partial differential equation (PDE). Oelz (2011) extended these methods to open curves. However, current theoretical analysis of curve-straightening flows is largely limited to single curves. In this work, we extend these methods to pairs of curves interacting with a molecular motor, and derive and analyse the associated partial differential equations.

The objectives of this paper are two-fold. First, we derive a PDE model for two semi-flexible filaments with a myosin motor attached at their intersection. Through this derivation, we describe how to obtain an explicit formula for the network stress tensor. We then use this model to investigate in detail how this two-filament structure facilitates microscopic-scale contraction. Since these dynamics are more complex than the single- 
curve straightening problem, we use a combination of asymptotic analysis and numerical solutions to achieve this. We apply the model to pairs of symmetric filaments with a motor initially attached to the minus-ends, and solve until the motor reaches the plus-ends and detaches. From this analysis, we propose a mechanism based neither on filament buckling, nor intrinsic force asymmetry where bending generates contraction. Instead, we find that filament semi-flexibility creates a geometric asymmetry that inhibits expansion. Two rigid filaments exhibit polarity-reversal symmetry, such that the contraction associated with a minus-end-located motor balances with expansion associated with a plus-end-located motor. Introducing filament bending breaks this symmetry, and brings the filaments closer to parallel as the motor approaches the plus-ends. This decreases the resistance to motion generated by the spring force through the motor, enabling it to move faster close to the plus-ends. This asymmetry inhibits expansive stress, and gives rise to net contraction on the microscopic scale.

\section{Mathematical Model}

We develop a mathematical model for a myosin motor attached to two overlapping actin filaments, as illustrated in Figure 2.1a. We represent filaments as open curves in $\mathbb{R}^{2}$, and denote their positions by $z_{i}(s(t), t)=\left(x_{i}(s(t), t), y_{i}(s(t), t)\right)$, for $i=1,2$. Here, $t$ is time and $s \in\left[0, L_{i}\right]$ is the arc length parameter, where $L_{i}$ is the length of the $i$-th filament. Since actin filaments are polarised, we adopt the convention that $s=0$ corresponds to the filament minus-end, and $s=L_{i}$ corresponds to the plus-end. We represent the myosin motor as a point object existing at the intersection between the two filaments. We track its position by introducing the variables $m_{i}(t) \in\left[0, L_{i}\right]$, such that $s=m_{i}$ is the position of the motor head attached to the $i$-th filament. We assume that no other proteins, for example cross-linkers, are present. The two-filament-motor system is located within a parallelogram-shaped domain, the adjacent sides of which are given by the vectors $\boldsymbol{L}_{x}=\left(L_{x x}, L_{x y}\right)^{T}$, and $\boldsymbol{L}_{y}=\left(L_{y x}, L_{y y}\right)$. The vectors $\boldsymbol{F}_{x}=\left(F_{x x}, F_{x y}\right)$ and $\boldsymbol{F}_{y}=\left(F_{y x}, F_{y y}\right)$, shown in Figure 2.1a, are the normal and shear force components acting on the domain boundaries. These can be obtained as part of the solution, and we will describe the method to compute these later.

We express the mathematical model for the filament and motor mechanics as a system of force-balance equations. In abstract terms, we write

$$
F_{a, \text { drag }}-\delta E_{a, \text { bend }}-\delta E_{a, \text { stretch }}-\delta E_{m, \text { stretch }}+F_{m, a}=\mathbf{o .}
$$

The first three terms in (2.1) describe the drag, bending, and longitudinal stretching forces respectively on actin filaments. The fourth term represents longitudinal stretching along the myosin motor, and the final term describes forces between filaments and motors. 


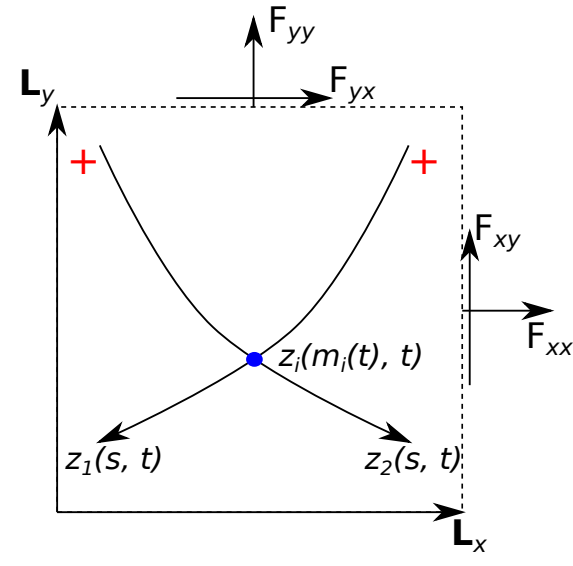

(a)

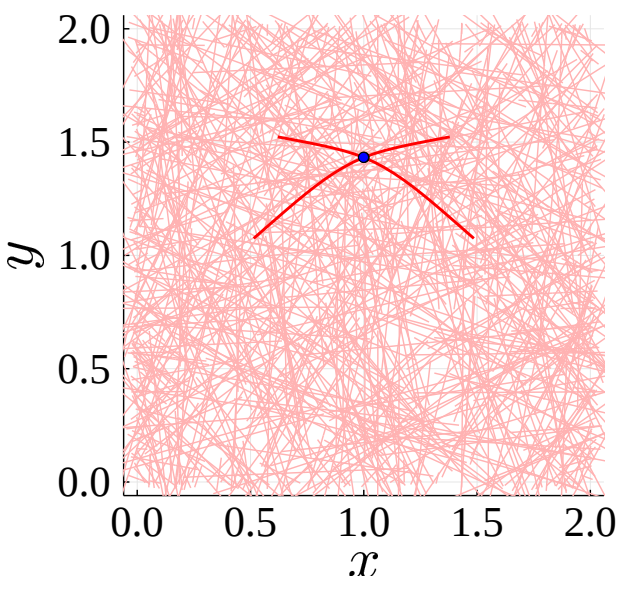

(b)

Figure 2.1: (a) Schematic representation of a two-filament-motor system. The filaments are the curves $z_{1}$ and $z_{2}$, and arrow heads represent filament minus (pointed) ends. Myosin motor proteins are represented by blue dots, and initially appear at the intersection between the two filaments. (b) A two-filament-motor structure immersed in a dense background network.

We represent bending and stretching forces as the variation of potential energy, where terms involving $\delta$ denote variations. We conceptualise drag as the combined effects of friction between the filaments and the cytoplasm, and protein friction (Tam, Mogilner, and Oelz 2021; McFadden et al. 2017; Bormuth et al. 2009; Tawada and Sekimoto 1991) acting at intersections between the two filaments and filaments in a background network. Figure 2.1b illustrates this scenario. In the limit of infinite background network density, protein friction acts uniformly along the two filaments in consideration, and represents the dominant contribution to overall drag.

\subsection{Energy Minimisation and Calculation of Stress}

To derive and simulate the model, we formulate the force-balance equations (2.1) as an energy minimisation problem. This involves constructing a time-discrete functional that sums the contribution of each force term,

$$
E\left[z_{1}, z_{2}, m_{1}, m_{2}\right]:=E_{a, \mathrm{drag}}+E_{a, \text { bend }}+E_{a, \text { stretch }}+E_{m, \text { stretch }}+E_{m, a} .
$$

In (2.2), $E_{a \text {,bend }}, E_{a \text {,stretch }}$ and $E_{m, \text { stretch }}$ are the potential energies associated with filament bending, filament stretching, and motor stretching respectively. The terms $E_{a, d r a g}$ and $E_{m, a}$ are pseudo-energy terms with variations that correspond to finite-difference approximations of $F_{a, \text { drag }}$ and $F_{m, a}$, which cannot be interpreted as variations of potential energy. At each time step of the simulation, the filament and motor positions are the minimiser of the functional (2.2). We outline each term in (2.2) below. 
The first term, $E_{a, \text { drag }}$, describes drag friction that opposes filament motion. Drag acts uniformly along the filaments, and could arise from viscous drag between filaments and the cytoplasm, or from protein friction between the filaments and a dense background network. The pseudo-energy term to represent this is

$$
E_{a, \mathrm{drag}}=\lambda_{a} \sum_{i=1}^{2} \int_{0}^{L_{i}} \frac{\left|z_{i}-\boldsymbol{F} z_{i}^{n}\right|^{2}}{2 \Delta t} \mathrm{~d} s,
$$

where $\lambda_{a}$ is the filament drag coefficient, $\Delta t$ is the time step size, and the superscript $n$ refers to the previous time step, i.e. $z_{i}^{n}=z_{i}(s, t-\Delta t)$. To account for possible stretching and rotation of the domain over time, we introduce the deformation gradient tensor,

$$
\boldsymbol{F}=\left[\begin{array}{ll}
L_{x x} / L_{x x}^{n} & L_{y x} / L_{y y}^{n} \\
L_{x y} / L_{x x}^{n} & L_{y y} / L_{y y}^{n}
\end{array}\right]
$$

such that multiplying $z_{i}^{n}$ by $\boldsymbol{F}$ ensures that $z_{i}$ and $z_{i}^{n}$ are both represented in the current spatial co-ordinates.

We model bending of semi-flexible actin filaments via the elastic potential energy

$$
E_{a, \text { bend }}=\sum_{i=1}^{2} \int_{0}^{L_{i}} \frac{\kappa_{a}}{2}\left|z_{i}^{\prime \prime}\right|^{2} \mathrm{~d} s
$$

where $\kappa_{a}$ is the flexural rigidity, and primes denote differentiation with respect to arc-length, $s$. We assume that $\kappa_{a}$ is constant, and the same for both filaments. We obtain a term for filament stretching by assuming that actin filaments are inextensible. To model this, we ensure that $\left|z_{i}^{\prime}\right|=1$ at every point along the filaments using the penalisation term

$$
E_{a, \text { stretch }}=\sum_{i=1}^{2} \int_{0}^{L_{i}} \frac{1}{\delta_{a}}\left(\left|z_{i}^{\prime}\right|-1\right)^{2} \mathrm{~d} s
$$

where $\delta_{a}$ is an arbitrarily small parameter that enforces the inextensibility constraints.

The remaining two terms in (2.2) describe how motors contribute to the mechanics. We ensure that motors remain point objects by introducing another penalising potential,

$$
E_{m, \text { stretch }}=\frac{1}{\delta_{m}}\left|z_{1}\left(m_{1}, t\right)-z_{2}\left(m_{2}, t\right)\right|^{2}
$$

where $\delta_{m}$ is an arbitrarily small parameter that penalises deviation from the constraint $z_{1}\left(m_{1}, t\right)=z_{2}\left(m_{2}, t\right)$. The final term in (2.2) describes interactions between filaments and motors. We assume that motors obey a linear (affine) force-velocity relationship. Subject to zero force, motors move with speed $V_{m}$. As the force through the motor increases, velocity decreases linearly until the motor stops, which occurs at the force $F_{s}$, known as the stall force. The corresponding pseudo-energy term consists of a linear term, and a 
quadratic drag-like term for the velocity reduction caused by the force through the motor,

$$
E_{m, a}=\sum_{j=1}^{2}\left(-F_{s} m_{j}+\frac{F_{s}}{V_{m}} \frac{\left(m_{j}-m_{j}^{n}\right)^{2}}{2 \Delta t}\right) .
$$

Minimising the functional (2.2) consisting of (2.3) and (2.5)-(2.8), then provides a timeimplicit numerical method to solve for the filament and motor positions.

Another advantage of energy minimisation is that it enables direct calculation of forces on the domain boundary that must be applied to prevent elongation and shear. These forces sum the contributions of both filaments and the motor, and provide a measure of net contractility. We obtain these forces $\boldsymbol{F}_{x}$ and $\boldsymbol{F}_{y}$ by adding extra terms to the energy functional, and defining

$$
E_{\text {total }}:=E+\boldsymbol{F}_{x} \cdot \boldsymbol{L}_{x}+\boldsymbol{F}_{y} \cdot \boldsymbol{L}_{y}
$$

If we impose that $\boldsymbol{L}_{x}$ and $\boldsymbol{L}_{y}$ remain constant, the vectors $\boldsymbol{F}_{x}=-\partial_{\boldsymbol{L}_{x}} E$ and $\boldsymbol{F}_{y}=-\partial_{\boldsymbol{L}_{y}} E$ represent Lagrange multipliers that enforce the constant domain size and shape constraints. After computing these, we describe the two-dimensional state of stress in the domain using the plane stress tensor,

$$
\boldsymbol{\sigma}=\left[\begin{array}{ll}
F_{x x} / L_{y y} & F_{x y} / L_{y y} \\
F_{y x} / L_{x x} & F_{y y} / L_{x x}
\end{array}\right]
$$

The bulk stress,

$$
\sigma=\frac{1}{2} \operatorname{tr}(\boldsymbol{\sigma})
$$

then provides a measure of the contraction or expansion generated by the two-filamentmotor system. By convention, negative $\sigma$ indicates contraction, and positive $\sigma$ indicates expansion. The quantity $\sigma$ is invariant to domain rotations, and equal to the average of the eigenvalues of $\boldsymbol{\sigma}$. The associated eigenvectors of $\boldsymbol{\sigma}$ are the principal stress directions, which indicate the directions of maximum contraction or expansion.

\subsection{Governing Equations}

Formulating the model as an energy minimisation problem enables us to derive PDEs that govern the filament and motor positions. The derivation is based on the following variational principle. It requires that, given known data $\left(z_{1}^{n}, z_{2}^{n}, m_{1}^{n}, m_{2}^{n}\right)$ at the discrete point in time $n$, the solution at the following point in time minimises the functional (2.2),

$$
\left(z_{1}^{n+1}, z_{2}^{n+1}, m_{1}^{n+1}, m_{2}^{n+1}\right)=\operatorname{argmin} E\left[z_{1}^{n}, z_{2}^{n}, m_{1}^{n}, m_{2}^{n}\right]\left(z_{1}, z_{2}, m_{1}, m_{2}\right)
$$


We obtain the corresponding force-balance equations by setting to zero the functional derivatives of (2.2) with respect to filament and motor positions. Subsequently, we write

$$
\delta E\left[z_{1}^{n}, z_{2}^{n}, m_{1}^{n}, m_{2}^{n}\right]\left(z_{1}^{n+1}, z_{2}^{n+1}, m_{1}^{n+1}, m_{2}^{n+1}\right) \cdot\left(\delta z_{1}, \delta z_{2}, \delta m_{1}, \delta m_{2}\right)=0,
$$

where terms involving $\delta$ denote the variation of the respective quantity. This formulation enables us to write (2.1) in terms of $z_{i}$ and $m_{i}$. We obtain the governing equations by evaluating (2.13) and matching coefficients of $\delta z_{1}, \delta z_{2}, \delta m_{1}$, and $\delta m_{2}$. On taking the formal continuum limit $\Delta t \rightarrow 0$, for which $\boldsymbol{F} \rightarrow \boldsymbol{I}$ and $\left(u-u^{n}\right) / \Delta t \rightarrow \dot{u}$, we obtain the system of PDEs

$$
\left\{\begin{array}{l}
\lambda_{a} \dot{z}_{1}+\kappa_{a} z_{1}^{\prime \prime \prime \prime}-\left(\lambda_{1} z_{1}^{\prime}\right)^{\prime}+\mu \frac{z_{1}-z_{2}}{\left\|z_{1}-z_{2}\right\|} \delta\left(s-m_{1}\right)=0, \\
\lambda_{a} \dot{z}_{2}+\kappa_{a} z_{2}^{\prime \prime \prime \prime}-\left(\lambda_{2} z_{2}^{\prime}\right)^{\prime}-\mu \frac{z_{1}-z_{2}}{\left\|z_{1}-z_{2}\right\|} \delta\left(s-m_{2}\right)=0, \\
\dot{m}_{1}=V_{m}\left[1-\frac{\mu}{F_{s}} \frac{z_{1}-z_{2}}{\left\|z_{1}-z_{2}\right\|} \cdot z_{1}^{\prime}\left(m_{1}, t\right)\right], \\
\dot{m}_{2}=V_{m}\left[1+\frac{\mu}{F_{s}} \frac{z_{1}-z_{2}}{\left\|z_{1}-z_{2}\right\|} \cdot z_{2}^{\prime}\left(m_{2}, t\right)\right],
\end{array}\right.
$$

where primes denote differentiation with respect to arc length, dots represent time derivatives, and $\delta(\cdot)$ is the Dirac delta function (not to be confused with variation). These equations (2.14) are a system of continuum force-balance equations for the filament and motor positions. They are formulated in a formal limit where $\delta_{a}$ and $\delta_{m}$ are small, and the force coefficients $1 / \delta_{a}$ and $1 / \delta_{m}$ in the variations of the penalising potentials (2.6) and (2.7) are replaced by the Lagrange multipliers $\lambda_{1}, \lambda_{2}$, and $\mu$. Note that the sign of $z_{1}-z_{2}$ in (2.14) will be absorbed by $\mu$. As a consequence, solutions satisfy the constraints

$$
\left\{\begin{array}{l}
\left|z_{i}^{\prime}\right| \equiv 1 \\
z_{1}\left(m_{1}, t\right)=z_{2}\left(m_{2}, t\right) .
\end{array}\right.
$$

The equations are subject to the boundary conditions

$$
\left\{\begin{array}{c}
z_{i}^{\prime \prime}(0, t)=z_{i}^{\prime \prime}\left(L_{i}, t\right)=0 \\
\left.\left(\kappa_{a} z_{i}^{\prime \prime \prime}-\lambda_{i} z_{i}^{\prime}\right)\right|_{s=0, L_{i}}=0
\end{array}\right.
$$

A detailed derivation of (2.14) and (2.16) is provided in Appendix A.

To obtain an expression for the bulk stress, $\sigma$, we differentiate the energy functional (2.9) 
with respect to $\boldsymbol{L}_{x}$ and $\boldsymbol{L}_{y}$. This yields

$$
\begin{aligned}
& \boldsymbol{F}_{x}=\lambda_{a} \sum_{i=1}^{2} \int_{0}^{L_{i}} \frac{x_{i}^{n}}{L_{x x}^{n}} \frac{\left(z_{i}-\boldsymbol{F} z_{i}^{n}\right)}{\Delta t} \mathrm{~d} s \\
& \boldsymbol{F}_{y}=\lambda_{a} \sum_{i=1}^{2} \int_{0}^{L_{i}} \frac{y_{i}^{n}}{L_{y y}^{n}} \frac{\left(z_{i}-\boldsymbol{F} z_{i}^{n}\right)}{\Delta t} \mathrm{~d} s .
\end{aligned}
$$

Then, applying the formal continuum limit $\Delta t \rightarrow 0, \boldsymbol{F} \rightarrow \boldsymbol{I},\left(u-u^{n}\right) / \Delta t \rightarrow \dot{u}$, and $z_{i}^{n} \rightarrow z_{i}$, we obtain

$$
\begin{aligned}
& \boldsymbol{F}_{x}=\lambda_{a} \sum_{i=1}^{2} \int_{0}^{L_{i}} \frac{x_{i}}{L_{x x}} \frac{\partial z_{i}}{\partial t} \mathrm{~d} s \\
& \boldsymbol{F}_{y}=\lambda_{a} \sum_{i=1}^{2} \int_{0}^{L_{i}} \frac{y_{i}}{L_{y y}} \frac{\partial z_{i}}{\partial t} \mathrm{~d} s .
\end{aligned}
$$

Evaluating the bulk stress (2.11) then yields

$$
\sigma=\lambda_{a} \sum_{i=1}^{2} \int_{0}^{L_{i}} \frac{x_{i}}{L_{x x}} \frac{\partial x_{i}}{\partial t}+\frac{y_{i}}{L_{y y}} \frac{\partial y_{i}}{\partial t} \mathrm{~d} s=\lambda_{a} \sum_{i=1}^{2} \int_{0}^{L_{i}} z_{i} \cdot \frac{\partial z_{i}}{\partial t} \mathrm{~d} s
$$

Furthermore, the expressions (2.18) confirm that

$$
\begin{aligned}
\sigma_{x y}-\sigma_{y x} & =\lambda_{a} \sum_{i=1}^{2} \int_{0}^{L_{i}} \frac{x_{i}}{L_{x x} L_{y y}} \frac{\partial y_{i}}{\partial t}-\frac{y_{i}}{L_{x x} L_{y y}} \frac{\partial x_{i}}{\partial t} \mathrm{~d} s \\
& =\frac{\lambda_{a}}{L_{x x} L_{y y}} \sum_{i=1}^{2} \int_{0}^{L_{i}}\left[\begin{array}{c}
-y_{i} \\
x_{i}
\end{array}\right] \cdot \frac{\partial z_{i}}{\partial t} \mathrm{~d} s \\
& =\frac{\lambda_{a}}{L_{x x} L_{y y}} \sum_{i=1}^{2} \int_{0}^{L_{i}} z_{i}^{\perp} \cdot \frac{\partial z_{i}}{\partial t} \mathrm{~d} s=0,
\end{aligned}
$$

We nondimensionalise the PDE model (2.14)-(2.16) by introducing the length and time scales

$$
\hat{t}=\frac{F_{s}}{\lambda_{a} L_{a}^{2}} t, \quad \text { and } \quad(\hat{x}, \hat{y})=\frac{1}{L_{a}}(x, y)
$$


where hats represent dimensionless variables, and $L_{a}$ is a characteristic filament length. The dimensionless model is then (dropping hats for convenience)

$$
\left\{\begin{array}{l}
\dot{z}_{1}+\kappa^{*} z_{1}^{\prime \prime \prime \prime}-\left(\lambda_{1}^{*} z_{1}^{\prime}\right)^{\prime}+\mu^{*} \frac{z_{1}-z_{2}}{\left\|z_{1}-z_{2}\right\|} \delta^{*}\left(s-m_{1}\right)=0 \\
\dot{z_{2}}+\kappa^{*} z_{2}^{\prime \prime \prime \prime}-\left(\lambda_{2}^{*} z_{2}^{\prime}\right)^{\prime}-\mu^{*} \frac{z_{1}-z_{2}}{\left\|z_{1}-z_{2}\right\|} \delta^{*}\left(s-m_{2}\right)=0 \\
\frac{1}{V_{m}^{*}} \dot{m}_{1}=1-\mu^{*} \frac{z_{1}-z_{2}}{\left\|z_{1}-z_{2}\right\|} \cdot z_{1}^{\prime}\left(m_{1}, t\right) \\
\frac{1}{V_{m}^{*}} \dot{m}_{2}=1+\mu^{*} \frac{z_{1}-z_{2}}{\left\|z_{1}-z_{2}\right\|} \cdot z_{2}^{\prime}\left(m_{2}, t\right)
\end{array}\right.
$$

subject to the boundary conditions

$$
\left\{\begin{array}{l}
z_{i}^{\prime \prime}(0, t)=z_{i}^{\prime \prime}\left(L_{i}^{*}, t\right)=0 \\
\left.\left(\kappa^{*} z_{i}^{\prime \prime \prime}-\lambda_{i}^{*} z_{i}^{\prime}\right)\right|_{s=0, L_{i}^{*}}=0
\end{array}\right.
$$

and the constraints

$$
\left\{\begin{array}{l}
\left|z_{i}^{\prime}\right| \equiv 1 \\
z_{1}\left(m_{1}, t\right)=z_{2}\left(m_{2}, t\right)
\end{array}\right.
$$

where $\delta^{*}(\hat{x})=L_{a} \delta\left(L_{a} \hat{x}\right)$ is a scaled Dirac delta function. The dimensionless parameters and forces are

$$
\kappa^{*}=\frac{\kappa}{F_{s} L_{a}^{2}}, \quad \lambda_{i}^{*}=\frac{\lambda_{i}}{F_{s}}, \quad \mu_{i}^{*}=\frac{\mu_{i}}{F_{s}}, \quad V_{m}^{*}=\frac{V_{m} \lambda_{a} L_{a}}{F_{s}}, \quad \text { and } \quad L_{i}^{*}=\frac{L_{i}}{L_{a}} .
$$

In subsequent sections, we consider asymptotic analysis and numerical solutions to the dimensionless system (2.22) and (2.23).

\subsection{Symmetric Filament-Motor Assemblies}

Before proceeding with the analysis, we consider a simplification to (2.22) and (2.23). First, we assume that the two filaments are symmetric about the vertical, that is

$$
z_{1}=z, \quad z_{2}=\left[\begin{array}{cc}
-1 & 0 \\
0 & 1
\end{array}\right] z
$$

and have identical length $L_{1}=L_{2}=L_{a}$. This symmetry also implies that the relative position of the motor is the same for both filaments, $m_{1}=m_{2}=m$, and that $\lambda_{1}^{*}=\lambda_{2}^{*}=\lambda^{*}$. To simplify the motor dynamics (2.22c) and (2.22d), we impose $V_{m} \rightarrow \infty$. Finally, we assume that filaments have large flexural rigidity $\kappa^{*}=1 / \varepsilon$, where $\varepsilon \ll 1$, and thus undergo small bending. On applying these simplifications, the dimensionless model (2.22) becomes 
(dropping asterisks on dimensionless parameters)

$$
\left\{\begin{array}{l}
\frac{\partial z}{\partial t}+\frac{1}{\varepsilon} z^{\prime \prime \prime \prime}-\left(\lambda z^{\prime}\right)^{\prime}+\mu\left(\begin{array}{l}
1 \\
0
\end{array}\right) \delta(s-m), \\
0=1-\mu\left(\begin{array}{l}
1 \\
0
\end{array}\right) \cdot z^{\prime}(m(t), t)
\end{array}\right.
$$

subject to the boundary and initial conditions

$$
\left\{\begin{array}{l}
z^{\prime \prime}(0, t)=z^{\prime \prime}(1, t)=0 \\
\left.\left(\frac{1}{\varepsilon} z^{\prime \prime \prime}-\lambda z^{\prime}\right)\right|_{s=0,1}=0 \\
z(s, t=0)=z_{I}(s)
\end{array}\right.
$$

and the constraints

$$
\left\{\begin{array}{l}
\left|z^{\prime}\right|=1, \\
z(m(t), t)=\left(\begin{array}{c}
0 \\
y(t)
\end{array}\right) .
\end{array}\right.
$$

The dimensionless form of the bulk stress (2.19) in the simplified model (2.27) and (2.28) is then

$$
\sigma=2 \int_{0}^{1} \frac{\partial z}{\partial t} \cdot z \mathrm{~d} s=-2 \int_{0}^{1} \frac{1}{\varepsilon}\left(z^{\prime \prime}\right)^{2}+\lambda \mathrm{d} s .
$$

To obtain a measure of net stress, we integrate $\sigma$ over the time between motor attachment and detachment. This yields

$$
\int_{0}^{T} \sigma \mathrm{d} t=J(T)-J(0)
$$

where

$$
J(t)=\int_{0}^{1}|z(s, t)|^{2} \mathrm{~d} s .
$$

The quantity $J(T)-J(0)$ describes the net, time-aggregated stress that the two filaments produce as they evolve. With this in mind, we use a combination of asymptotic analysis and numerical solutions to investigate the effect of filament bending on contraction. 


\section{Asymptotic Analysis}

We construct an asymptotic approximation to the solution of the model for the simplified symmetric problem (2.27)-(2.29). To do this, we expand variables in powers of $\varepsilon$,

$$
\begin{aligned}
z & =z_{0}+\varepsilon z_{1}+\varepsilon^{2} z_{2}+\mathcal{O}\left(\varepsilon^{3}\right), \\
m & =m_{0}+\varepsilon m_{1}+\varepsilon^{2} m_{2}+\mathcal{O}\left(\varepsilon^{3}\right), \\
\lambda & =\lambda_{0}+\varepsilon \lambda_{1}+\varepsilon^{2} \lambda_{2}+\mathcal{O}\left(\varepsilon^{3}\right), \\
\mu & =\mu_{0}+\varepsilon \mu_{1}+\varepsilon^{2} \mu_{2}+\mathcal{O}\left(\varepsilon^{3}\right), \\
\sigma & =\sigma_{0}+\varepsilon \sigma_{1}+\varepsilon^{2} \sigma_{2}+\mathcal{O}\left(\varepsilon^{3}\right), \\
J & =J_{0}+\varepsilon J_{1}+\varepsilon^{2} J_{2}+\mathcal{O}\left(\varepsilon^{3}\right),
\end{aligned}
$$

We obtain an ansatz for the leading-order solution for rigid filaments from the $\mathcal{O}(1 / \varepsilon)$ problem. On substituting the asymptotic expansions (3.1) into the simplified model (2.27)(2.29), at $\mathcal{O}(1 / \varepsilon)$ we obtain

$$
z_{0}^{\prime \prime \prime \prime}=0,\left.\quad z_{0}^{\prime \prime \prime}\right|_{s=0,1}=0,\left.\quad z_{0}^{\prime \prime}\right|_{s=0,1}=0, \quad\left|z_{0}^{\prime}\right|=1
$$

The solution to (3.2) for $z_{0}$ is a straight filament, whose direction we parameterise by the filament angle, $\theta / 2$, measured from the positive vertical axis (see Figure 3.1) writing

$$
z_{0}^{\prime}=\left[\begin{array}{l}
\sin (\theta / 2) \\
\cos (\theta / 2)
\end{array}\right] .
$$

Note that for the orthogonal direction we use the notation

$$
z_{0}^{\perp}=\left[\begin{array}{c}
-\cos (\theta / 2) \\
\sin (\theta / 2)
\end{array}\right]
$$




$$
z_{0}=\left(\begin{array}{c}
0 \\
y_{0}
\end{array}\right)+z_{0}^{\prime}\left(s-m_{0}\right),
$$

where the leading-order motor relative position, $m_{0}$, and leading-order vertical position of the intersection, $y_{0}$, complete the parameterisation. This situation is illustrated in Figure 3.1.

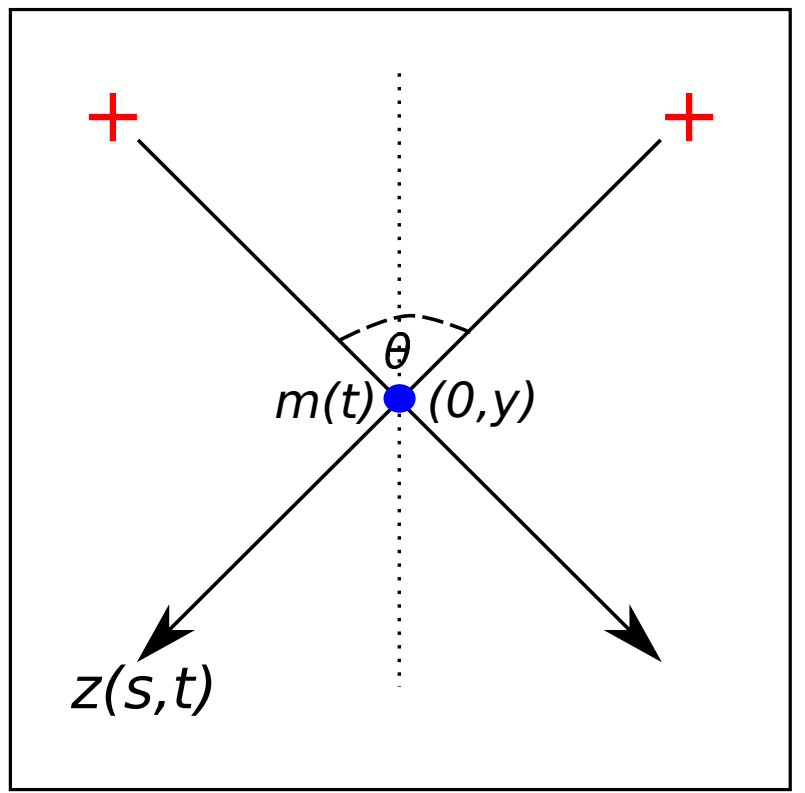

Figure 3.1: Schematic of the simplified two-filament-motor system with two rigid, symmetric actin filaments. The myosin motor has relative position $m(t)$, and physical position $(0, y(t))$. The two filaments are symmetric about the dashed vertical line, which is the positive $y$-axis. The angle between the filaments is $\theta$, such that the angle between a filament and the $y$-axis is $\theta / 2$. 


\subsection{Leading-Order Solution}

To obtain the leading-order solution, we consider the $\mathcal{O}(1)$ problem

$$
\left\{\begin{array}{l}
\frac{\partial z_{0}}{\partial t}+z_{1}^{\prime \prime \prime \prime}-\left(\lambda_{0} z_{0}^{\prime}\right)^{\prime}+\mu_{0}\left(\begin{array}{l}
1 \\
0
\end{array}\right) \delta\left(s-m_{0}\right)=0 \\
0=1-\mu_{0} \sin \left(\frac{\theta}{2}\right) \\
z_{1}^{\prime \prime \prime}-\left.\lambda_{0} z_{0}^{\prime}\right|_{s=0,1}=0 \\
z_{0}\left(m_{0}(t), t\right)=\left(\begin{array}{c}
0 \\
y_{0}(t)
\end{array}\right) \\
z_{0}^{\prime} \cdot z_{1}^{\prime}=0
\end{array}\right.
$$

To proceed, we use the orthogonality condition (3.6e) to infer the ansatz

$$
z_{1}^{\prime}(s, t)=h^{\prime}(s, t) z_{0}^{\prime \perp}
$$

where $h(s, t)$ is an arbitrary scalar function. Substituting (3.5) and (3.7) into (3.6a) enables us to solve for the leading-order quantities

$$
\mu_{0}=\frac{1}{\sin (\theta / 2)}, \quad \lambda_{0}=H\left(s-m_{0}\right)-s, \quad \text { and } \quad \sigma_{0}=2 \nu_{0}
$$

where $H$ is the Heaviside step function, and $\nu_{0}=m_{0}-1 / 2$. Filament evolution then satisfies the ordinary differential equations

$$
\begin{gathered}
\frac{\mathrm{d} S}{\mathrm{~d} t}=-24 \nu_{0}(1-S), \\
S \frac{\mathrm{d} \nu_{0}}{\mathrm{~d} t}=1+12 \nu_{0}^{2}(1-S),
\end{gathered}
$$

where $S=\sin ^{2}(\theta / 2)$. Since $z_{0}$ is written in terms of the angle $\theta$ only, the system (3.9) determines $z_{0}$, and since $h^{\prime}$ is known, we subsequently obtain $z_{1}$. Full details on this calculation are available in Appendix B.

An important property of the system (3.9) is that it is invariant under a change of variables which reverses the direction of time. If we introduce the reversed-time $\tilde{t}=T-t$ for an arbitrary constant $T$, we have $\tilde{\nu}_{0}(\tilde{t})=-\nu_{0}(T-\tilde{t})$ and $\tilde{\theta}(\tilde{t})=\theta(T-\tilde{t})(i . e . \quad \tilde{S}=S)$. Consequently, if the motor is initially positioned at the pointed ends $\left(\nu_{0}(0)=-1 / 2\right)$, and 
$T$ denotes the time it reaches the barbed ends, then the time-aggregated stress vanishes,

$$
\begin{aligned}
J(T)-J(0) & =\int_{0}^{T / 2} \sigma(t) \mathrm{d} t+\int_{T / 2}^{T} \sigma(t) \mathrm{d} t \\
& =\int_{0}^{T / 2} 2 \nu_{0}(t) \mathrm{d} t+\int_{0}^{T / 2} 2 \nu_{0}(T-\tilde{t}) \mathrm{d} \tilde{t} \\
& =\int_{0}^{T / 2} 2 \nu_{0} \mathrm{~d} t-\int_{0}^{T / 2} 2 \tilde{\nu}_{0} \mathrm{~d} \tilde{t}=0 .
\end{aligned}
$$

The higher-order correction terms, $z_{1}, \sigma_{1}$ and $J_{1}$, elucidate the effect of small, non-zero bending on filament evolution and stress. To solve for these terms, we substitute the asymptotic expansions (3.1) into (2.30) and (2.31) to obtain

$$
\begin{aligned}
& \sigma=-2 \int_{0}^{1} \lambda_{0} \mathrm{~d} s-2 \varepsilon \int_{0}^{1}\left(\left|z_{1}^{\prime \prime}\right|^{2}+\lambda_{1}\right) \mathrm{d} s+\mathcal{O}\left(\varepsilon^{2}\right) \\
& J=\int_{0}^{1}\left|z_{0}\right|^{2} \mathrm{~d} s+2 \varepsilon \int_{0}^{1} z_{0} \cdot z_{1} \mathrm{~d} s+\mathcal{O}\left(\varepsilon^{2}\right) .
\end{aligned}
$$

Matching coefficients of $\varepsilon$ then yields

$$
\sigma_{1}=-2 \int_{0}^{1}\left(\left|z_{1}^{\prime \prime}\right|^{2}+\lambda_{1}\right) \mathrm{d} s, \quad J_{1}=2 \int_{0}^{1} z_{0} \cdot z_{1} \mathrm{~d} s
$$

In addition, we use the PDE (3.6a) and the ansatz (3.7) to obtain an explicit expression for the curvature of $z_{1}$,

$$
h^{\prime \prime}=-\cot \left(\frac{\theta}{2}\right)\left[\left(m_{0}-s\right) H\left(s-m_{0}\right)+s^{2}\left(m_{0}(2 s-3)-s+2\right)\right] .
$$


Since the first-order correction to stress, $\sigma_{1}$, involves the currently unknown $\lambda_{1}$, progress requires consideration of the $\mathcal{O}(\varepsilon)$ problem, which is

$$
\left\{\begin{array}{l}
\frac{\partial z_{1}}{\partial t}+z_{2}^{\prime \prime \prime \prime}-\left(\lambda_{0} z_{1}^{\prime}+\lambda_{1} z_{0}^{\prime}\right)^{\prime}+\mu_{1}\left(\begin{array}{l}
1 \\
0
\end{array}\right) \delta\left(s-m_{0}\right)-\mu_{0}\left(\begin{array}{l}
1 \\
0
\end{array}\right) \delta^{\prime}\left(s-m_{0}\right) m_{1}=0 \\
0=-\left(\begin{array}{l}
1 \\
0
\end{array}\right)\left(\mu_{1} z_{0}^{\prime}\left(m_{0}(t), t\right)+\mu_{0} z_{1}^{\prime}\left(m_{0}(t), t\right)\right) \\
z_{2}^{\prime \prime \prime}-\lambda_{0} z_{1}^{\prime}-\left.\lambda_{1} z_{0}^{\prime}\right|_{s=0,1}=0 \\
\left.z_{2}^{\prime \prime}\right|_{s=0,1}=0 \\
z_{1}\left(m_{0}(t), t\right)+z_{0}^{\prime}\left(m_{1}(t), t\right)=\left(\begin{array}{c}
0 \\
y_{1}(t)
\end{array}\right) \\
\left|z_{1}^{\prime}\right|^{2}+2 z_{0}^{\prime} \cdot z_{2}^{\prime}=0 .
\end{array}\right.
$$

Obtaining the solution to (3.14) involves an intricate calculation based on the ansatz

$$
z_{1}=\left(\begin{array}{c}
0 \\
y_{1}(t)
\end{array}\right)-z_{0}^{\prime}(t) m_{1}+z_{0}^{\perp \perp}\left[A(t)\left(s-m_{0}\right)+\int_{m_{0}}^{s} \tilde{h}^{\prime}(s, t) \mathrm{d} s\right]
$$

which reflects (3.14e) and (3.7). This gives rise to a system of equations for the degrees of freedom $A(t), y_{1}(t)$, and $m_{1}(t)$. We provide full details on the calculation to obtain this in Appendix B. A key result is the stress correction term,

$$
\sigma_{1}=-2 \int_{0}^{1}\left|h^{\prime \prime}\right|^{2} \mathrm{~d} s-2\left[A+\tilde{h}^{\prime}\left(m_{0}, t\right)\right] \cot \left(\frac{\theta}{2}\right)\left(\frac{1}{2}-m_{0}\right)+2 m_{1}
$$

where $\tilde{h}^{\prime}\left(m_{0}, t\right)$ is given by

$$
\tilde{h}^{\prime}\left(m_{0}, t\right)=-\frac{1}{12} m_{0}^{3}\left(6 m_{0}^{2}-15 m_{0}+8\right) \cot \left(\frac{\theta}{2}\right) .
$$

Similar to the system (3.9), we can obtain a system of differential equations to solve for $A(t), y_{1}(t)$, and $m_{1}(t)$. Since $h^{\prime \prime}$ and $\tilde{h}^{\prime}$ are in terms of the leading-order degrees of freedom $\theta$ and $m_{0}$, we can subsequently compute $\sigma_{1}$. However, the ODEs for $A(t), y_{1}(t)$, and $m_{1}(t)$ have no exact solution. Therefore, we continue our investigation using numerical solutions.

\section{Numerical Solutions}

We compute numerical solutions to the simplified system (2.27) in JULIA, by constructing a dimensionless version of the functional (2.2), and using Optim.jl (Mogensen and Risbeth 2018) to obtain the minimiser at each time step. This is equivalent to a time-implicit 
numerical method for solving (2.22). We represent filaments as 50 equal-length line segments joined at nodes, about which segments can rotate. Each filament has total length $1 \mu \mathrm{m}$ (Kamasaki, Osumi, and Mabuchi 2007), and we solve the model using the time step size $\Delta t=0.001$. We perform the minimisation using the limited-memory BroydenFletcher-Goldfarb-Shanno (LBFGS) method and incorporate automatic differentiation (ForwardDiff.jl) to compute the gradient of the energy (2.2).

\subsection{Comparison With Asymptotic Analysis}

We begin by computing numerical solutions for two symmetric filaments with $m(0)=0$, and $\theta(0)=\pi / 2$. Like the asymptotic analysis, we assume these filaments are initially rigid, $V_{m} \rightarrow \infty$, and solve until the motor reaches the plus-end and detaches. First, we compute a solution for two rigid $\left(\varepsilon=1 \times 10^{-5}\right)$ filaments, to validate the leading-order bulk stress $\sigma_{0}=2 \nu_{0}$, and the solution to (3.9) which governs $z_{0}$. As Figures 4.1a and 4.1b show, for both of these we obtain agreement between the numerical solution and leading-order solution. Furthermore, Figure 4.1c illustrates the result from (3.10), namely that zero net stress is generated when a motor traverses two rigid filaments from the minus to plus-ends, i.e. $J_{0}(T)=J_{0}(0)=0$, where $T=0.627$ is the time at which the motor reaches the plus end.

Next, we solve the model with $\varepsilon=0.01$ to validate the formulae for $h^{\prime \prime}$ and $\sigma_{1},(3.13)$ and (3.16) respectively. The dynamics of the two filaments and motor are illustrated in Figure 4.2. As part of the solution, we compute $h^{\prime \prime}$ using the asymptotic formula (3.13) and numerical values of $\theta$ and $m$, and compare this with the numerical value for the curvature,

$$
h^{\prime \prime}=\frac{1}{\varepsilon}\left(z_{0}^{\prime \perp} \cdot z^{\prime \prime}\right) .
$$

This comparison is shown in Figure 4.3. At each time step, we obtain agreement between the numerical and asymptotic results. The curvature formula (3.13) also reveals the shape that the two filaments adopt as they evolve (the qualitative pattern is easier to see in Figure 4.6). Initially, the filaments adopt a convex shape, as the positive curvature in Figure 4.3a shows. As the motor moves and pulls the filaments inwards, their shape changes to concave, as Figures $4.3 \mathrm{c}$ and $4.3 \mathrm{~d}$ show. When the motor approaches the plus-end, the filaments return to a convex shape. The asymptotic result for $h^{\prime \prime}$ remains accurate for up to $\varepsilon \sim \mathcal{O}(1)$, before breaking down for $\varepsilon \sim \mathcal{O}(10)$.

We also use the numerical solution with $\varepsilon=0.01$ to validate the formula for $\sigma_{1}$, the first-order correction to bulk stress. At each time step, we compute the stress $\sigma$, and compare with the stress in a simulation with $\varepsilon=1 \times 10^{-4}$, which we consider to be $\sigma_{0}$ for rigid filaments. We then approximate the first-order correction as $\sigma_{1} \approx\left(\sigma-\sigma_{0}\right) / \varepsilon$, and present results in Figure 4.4a. For most values of $t$, it holds that $\sigma_{1}>0$. In particular, larger positive values of $\sigma_{1}$ occur close to $t=0$ and $t=T$, or $m=0$ and $m=1$. 


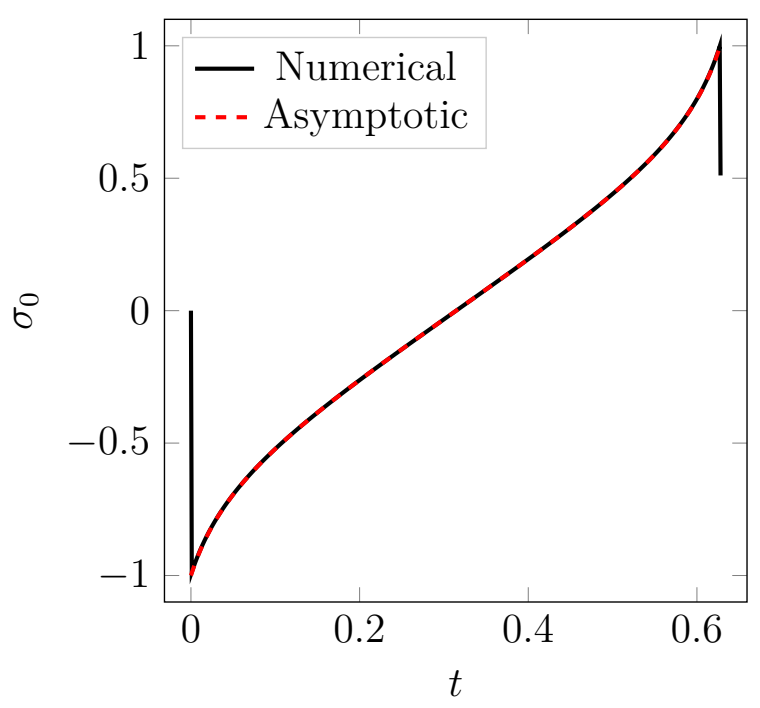

(a) Leading-order bulk stress, $\sigma_{0}$.

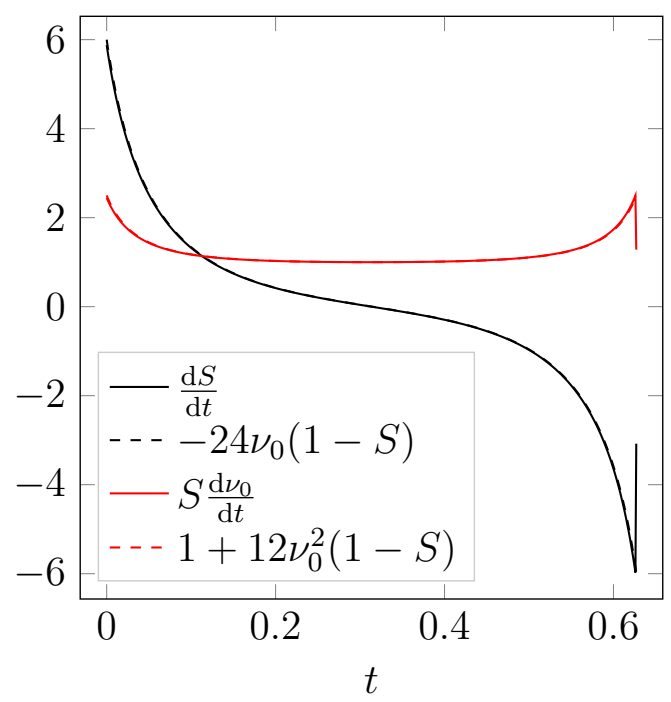

(b) System of ODEs, (3.9).

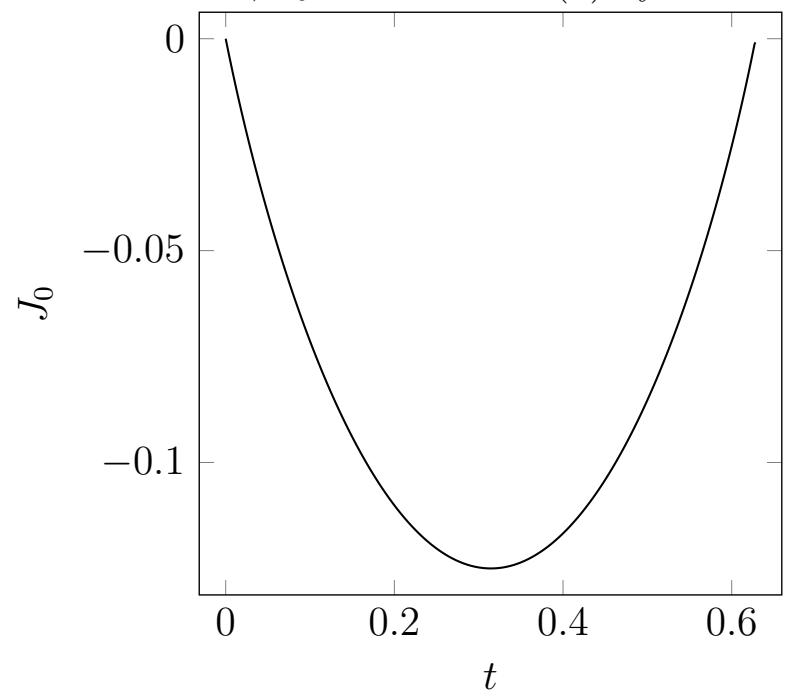

(c) $J_{0}$

Figure 4.1: Comparison between a numerical solution with rigid filaments $\left(\varepsilon=1 \times 10^{-5}\right)$ and the leading-order asymptotic solution. 


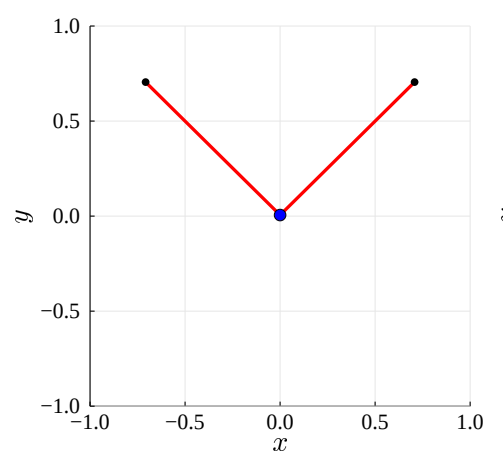

(a) $t=0.001$.

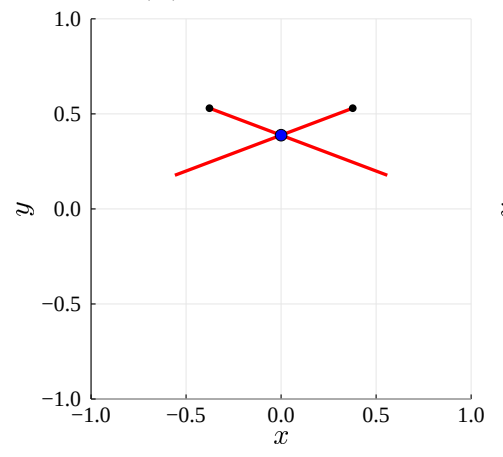

(d) $t=0.4$.

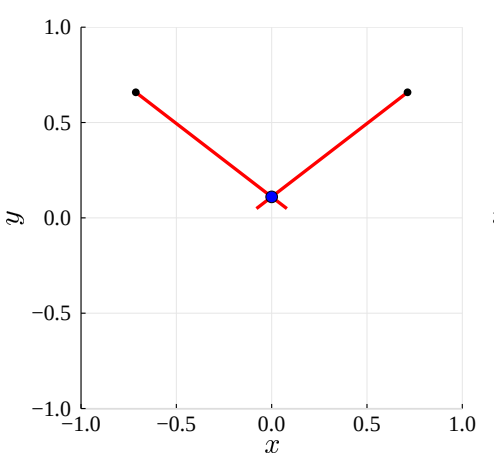

(b) $t=0.028$.

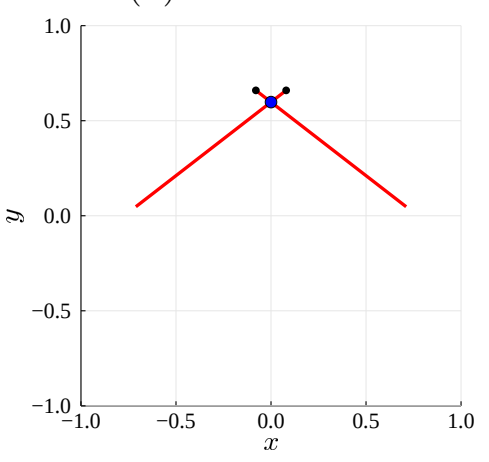

(e) $t=0.6$.

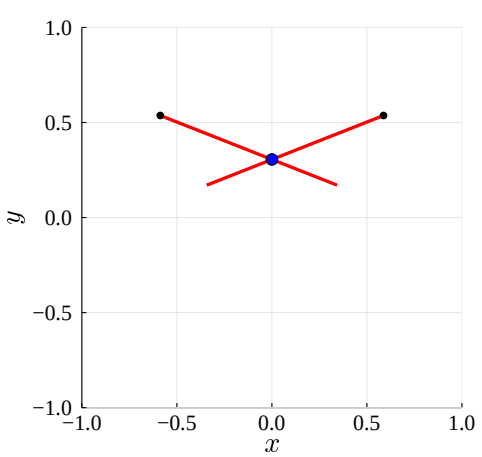

(c) $t=0.2$.

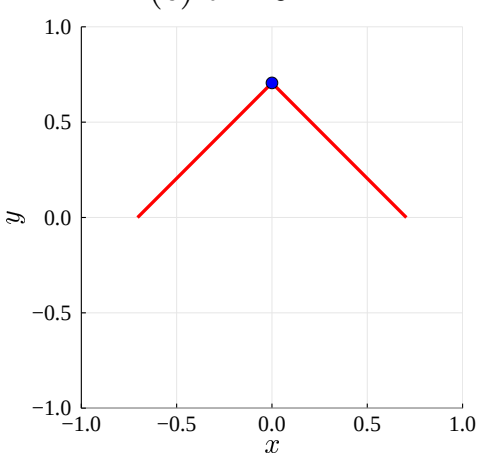

(f) $t=0.627$.

Figure 4.2: Numerical solution for the evolution of two actin filaments (red solid curves) with $\varepsilon=0.01$. The black nodes indicate the filament plus ends, and the blue dot at the filament intersection represents the myosin motor.

Figure 4.4a is surprising, because it suggests the introduction of filament bending generates stresses that are biased to expansion. Similarly, as Figure 4.4b shows, the quantity $J_{1}(T)-J_{1}(0)>0$, also suggesting net expansive bias. Based on this, one might conclude that bending cannot facilitate microscopic-scale contraction. However, we have not yet accounted for the changes in filament geometry, and how they influence motor dynamics. Further simulations in $\$ 4.2$ will reveal this more clearly, and confirm that bending does facilitate net microscopic-scale contraction.

\subsection{Symmetric Flexible Filaments}

We now consider numerical solutions beyond the $\varepsilon \ll 1$ regime considered in the asymptotic analysis. These solutions are with the same conditions as Figure 4.2, where the motor is initially at the minus-ends of two symmetric filaments. We then solve the model until the motor reaches the plus-ends. Results are presented in Figure 4.5. The quantity $J(t)$ measures the effect of $\varepsilon$ on net stress. This is shown in Figure 4.5a. For rigid filaments, we showed that $J(T)-J(0)=0$, indicating zero net stress as the motor moved from the minus to the plus-ends. Since $J(T)$ decreases as $\varepsilon$ increases, the introduction of filament bending facilitates bias to contraction. This is despite the quantities $\sigma_{1}(T)$ and $J_{1}(T)$ being positive, as in Figure 4.4a and 4.4b. Indeed, Figure 4.5b confirms that $\sigma_{1}>0$, with 

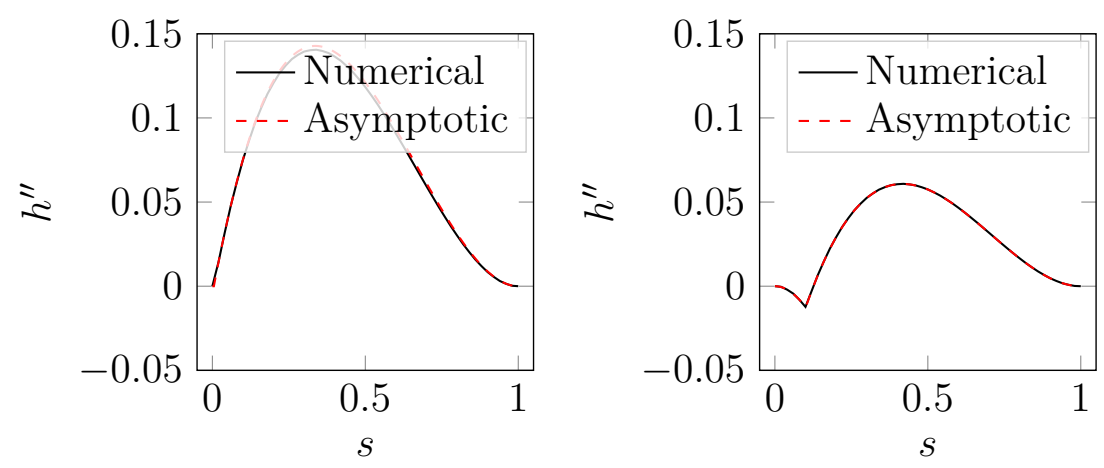

(a) $t=0.001$.

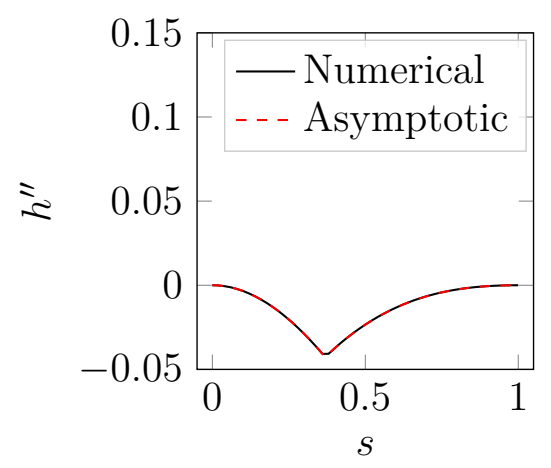

(b) $t=0.028$.

(c) $t=0.2$.
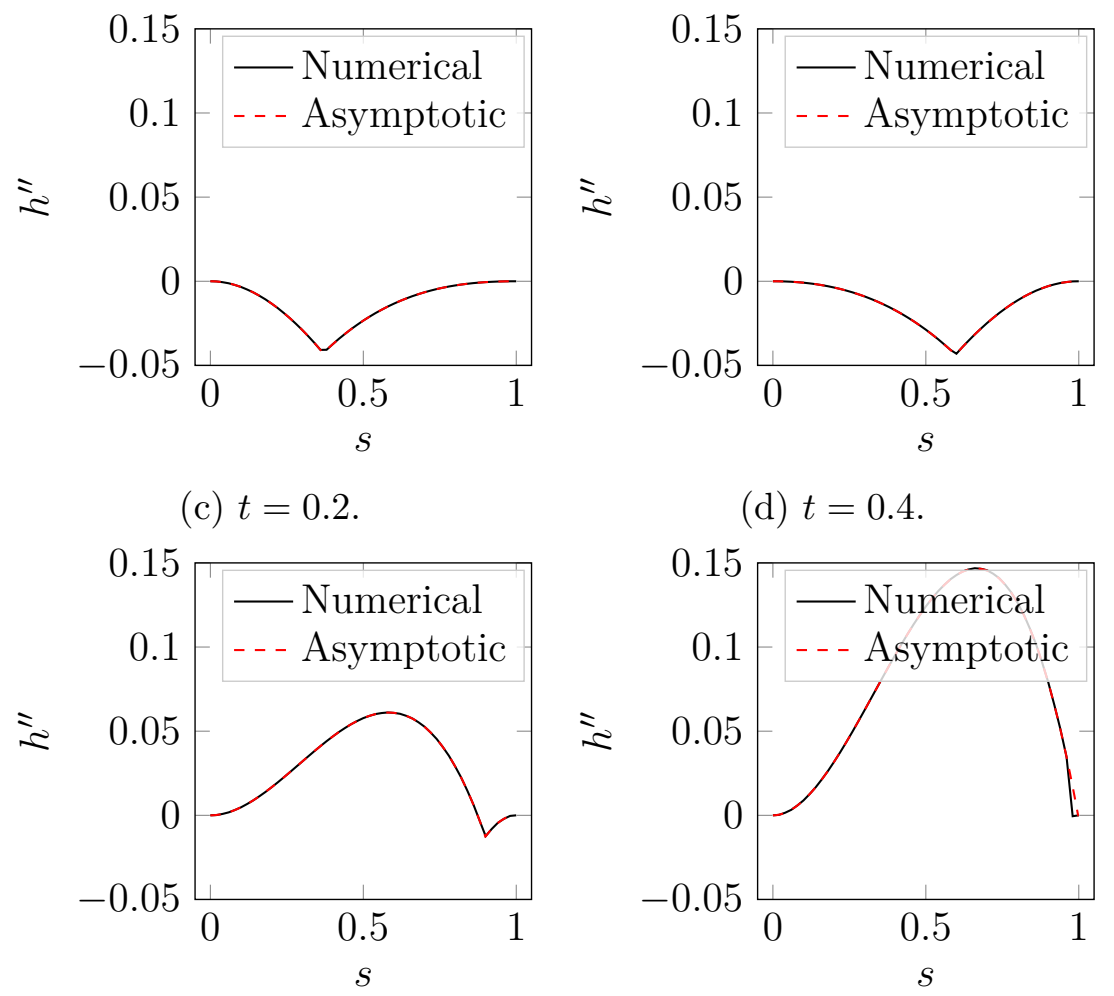

(d) $t=0.4$.

(e) $t=0.6$.

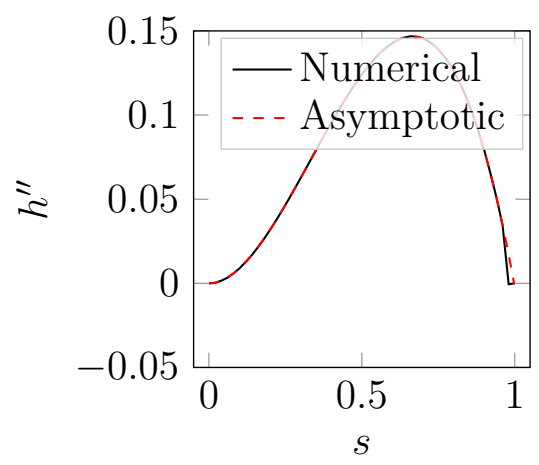

(f) $t=0.627$.

Figure 4.3: Numerical and asymptotic solutions for $h^{\prime \prime}(s, t)$, the curvature of $z_{1}$, in a numerical solution with $\varepsilon=0.01$, and $\theta(0)=\pi / 2$. 


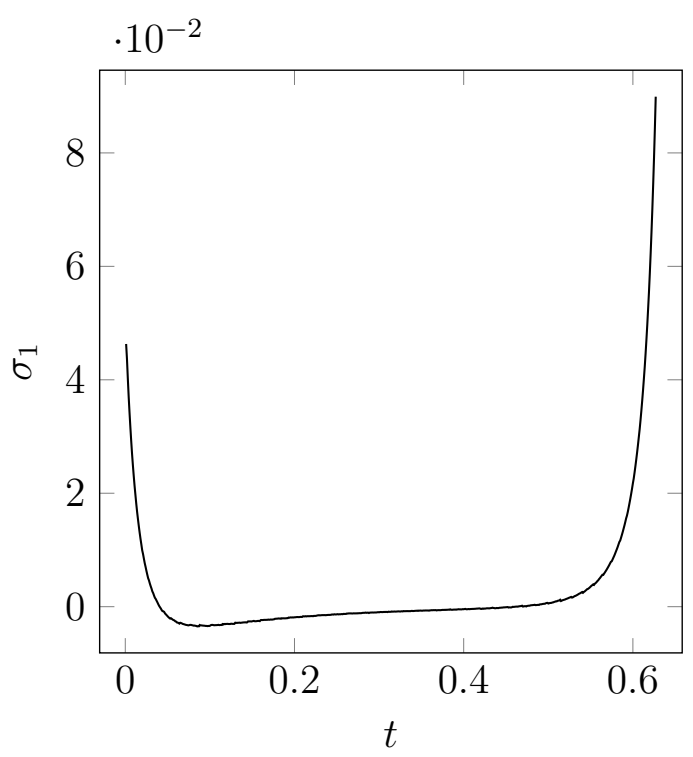

(a) $\sigma_{1}$

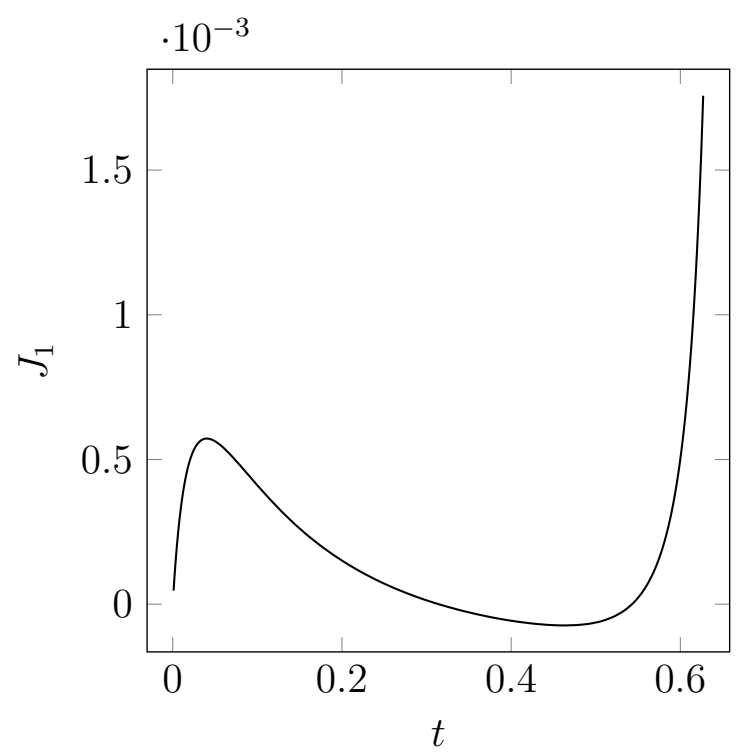

(b) $J_{1}$

Figure 4.4: Calculation of $\sigma_{1} \approx\left(\sigma-\sigma_{0}\right) / \varepsilon$ and $J_{1} \approx\left(J-J_{0}\right) / \varepsilon$ in the numerical solution with $\varepsilon=0.01$. The values of $\sigma_{0}$ and $J_{0}$ were obtained using a solution with $\varepsilon=1 \times 10^{-4}$.

stress increasing with $\varepsilon$ close to $t=0$ and $t=T$.

Semi-flexible filaments facilitates net contraction because bending breaks the polarityreversal symmetry, and the resulting geometry favours contraction. As Figure 4.5c shows, with increasing $\varepsilon$, the myosin motor moves faster along the filaments and detaches earlier. The increase in motor speed is largest as the motor approaches the plus-ends, which Figure $4.5 \mathrm{~b}$ shows is associated with expansion. As the motor approaches the plus-ends, the semi-flexible filaments adopt a convex shape that brings them closer to parallel at their tips, as illustrated in Figure 4.5d. This decreases the spring force through the motor, enabling it to move faster. Since the motor moves faster close to the plus-ends, the expansive component persists for shorter time than the contractile component. Consequently, the time-integrated stress $J(T)-J(0)$ decreases as $\varepsilon$ increases.

The results in Figure 4.5 are relevant for in vivo actin filaments, for which the parameters (Kamasaki, Osumi, and Mabuchi 2007; Gittes et al. 1993; Thoresen, Lenz, and Gardel 2011; Reichl et al. 2008; Oelz, Rubinstein, and Mogilner 2015) estimated in Tam, Mogilner, and Oelz (2021) give $\varepsilon=68.5$. To further our analysis, we compute a numerical solution with $\varepsilon=68.5$ and $V_{m}=1$, to investigate whether contraction persists after relaxing the assumption of infinite motor velocity. The evolution of these filaments is shown in Figure 4.6. Despite the slower motor speed, the evolution qualitatively follows the prediction from Figure 4.3. Filaments are initially convex, then become concave, and adopt a convex shape again as the motor approaches the plus-ends. As Figure 4.6f shows, the two filaments are curved when the motor reaches the plus-ends and detaches. To rule out the possibility that relaxation to straight configuration produces expansion that cancels out net contraction, we continued the simulation after motor detachment, until the 


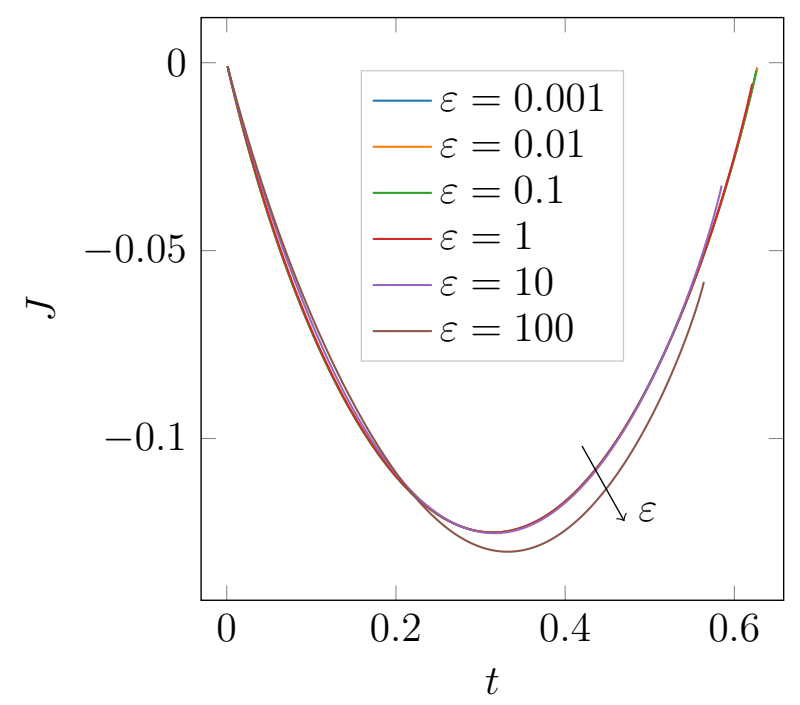

(a) $J(t)$

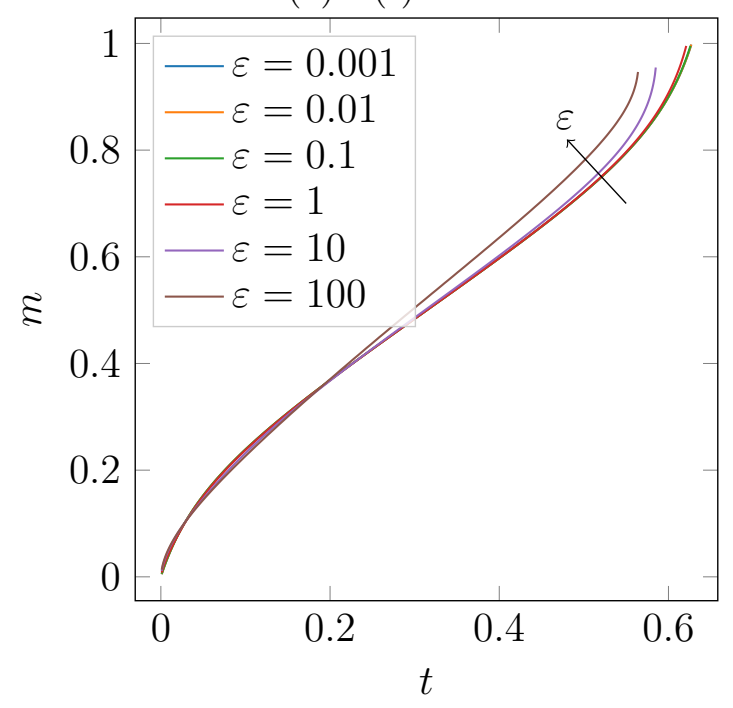

(c) Motor position, $m(t)$.

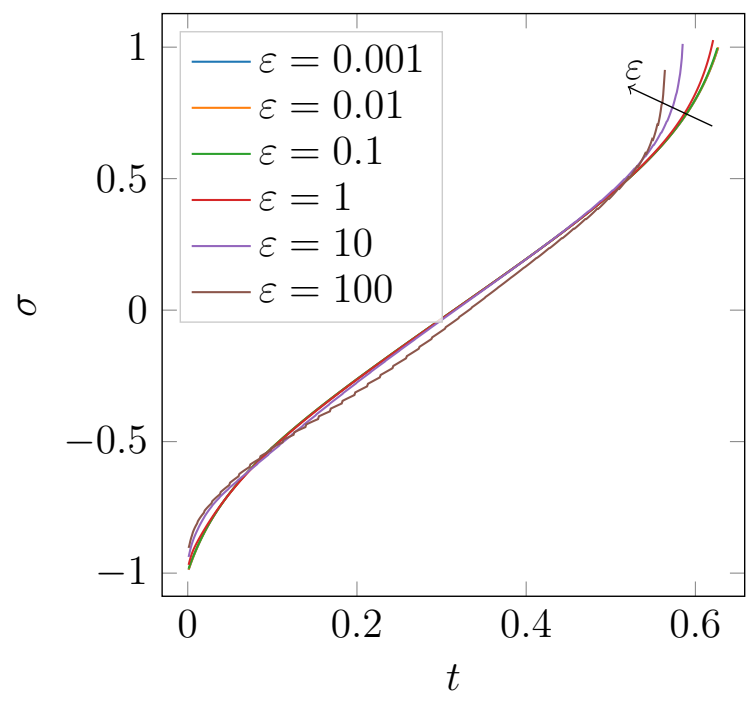

(b) Bulk stress, $\sigma$.

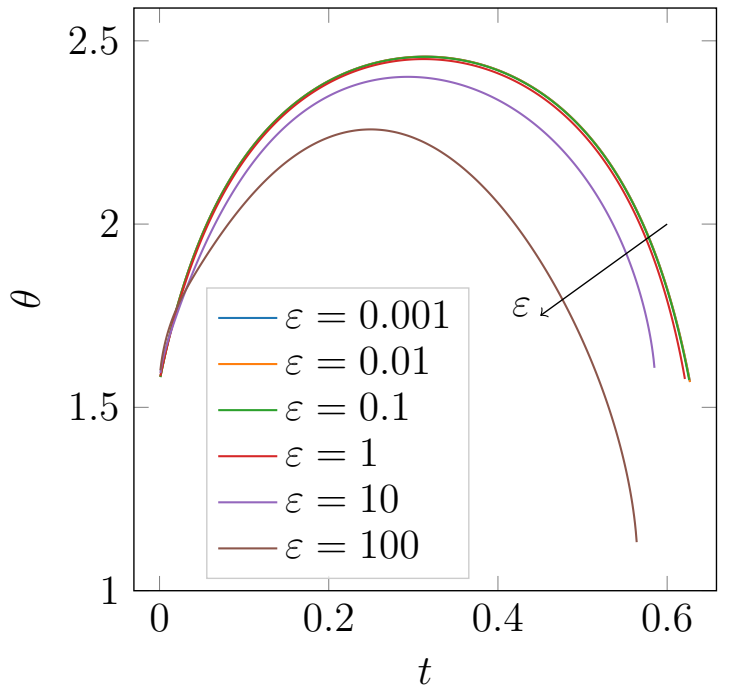

(d) Mean angle, $\theta$.

Figure 4.5: The effect of $\varepsilon$ on relevant quantities in solutions of two symmetric filaments. Solutions are computed with $m(0)=0, \theta(0)=\pi / 2$, and proceed for all $t \in[0, T(\varepsilon)]$ such that $m(t)<1$. After this time $T$, the motor reaches the plus-end and detaches. Results are plotted for six values of $\varepsilon$, and arrows indicate the direction of increasing $\varepsilon$. 


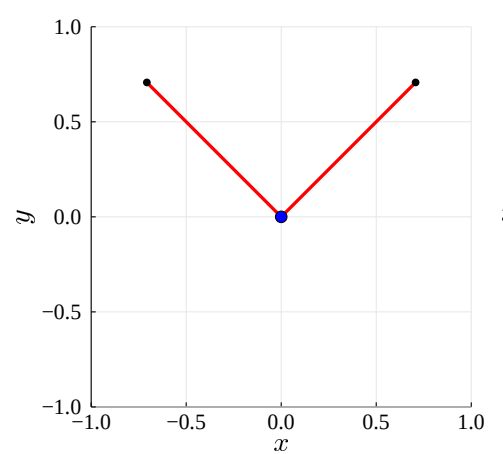

(a) $t=0$.

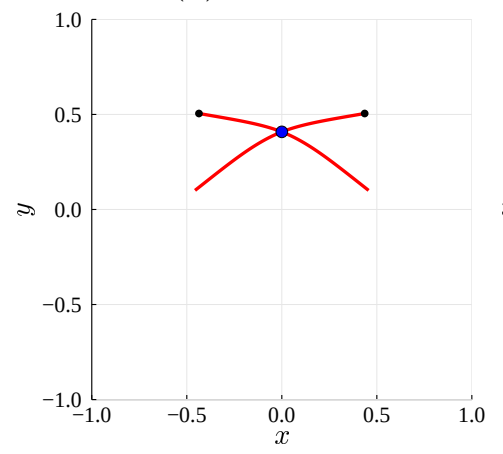

(d) $t=0.9$.

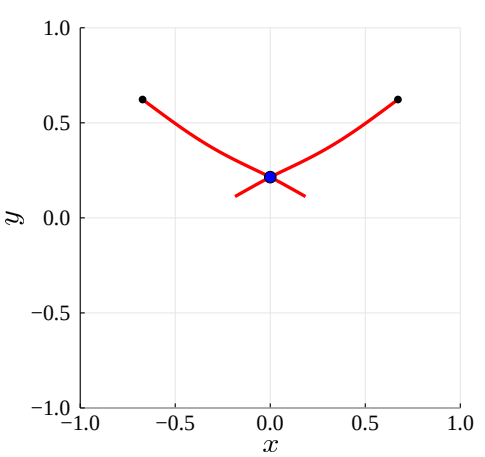

(b) $t=0.3$.

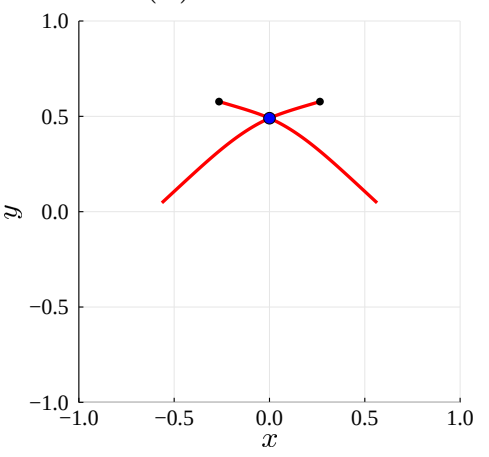

(e) $t=1.2$.

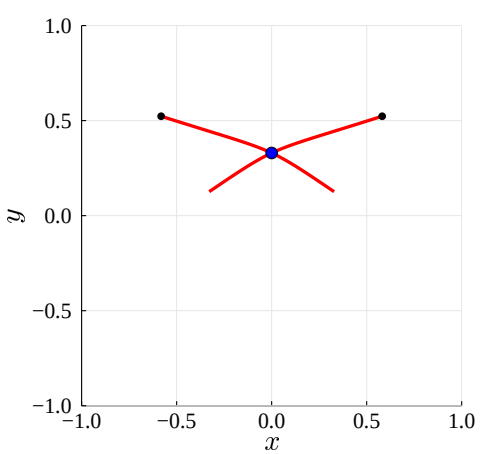

(c) $t=0.6$.

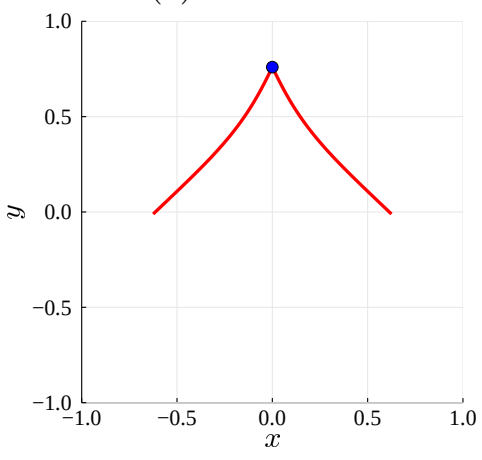

(f) $t=1.567$.

Figure 4.6: Numerical solution for the evolution of two flexible actin filaments (red solid curves) with $\varepsilon=68.5$ and $V_{m}=1$. The black nodes indicate the filament plus ends, and the blue dot at the filament intersection represents the myosin motor.

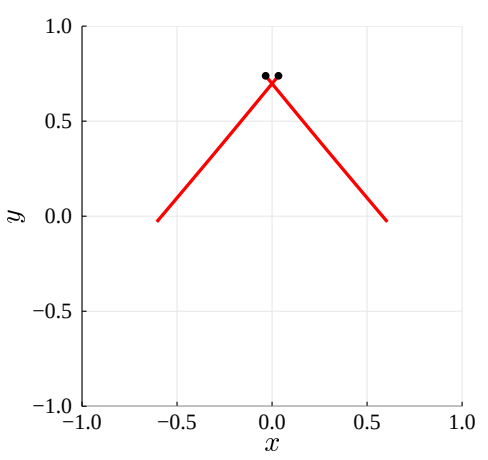

(a) Solution at $t=2$.

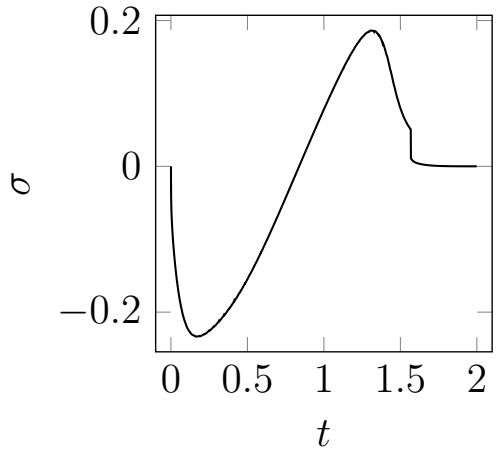

(b) Bulk stress, $\sigma$.

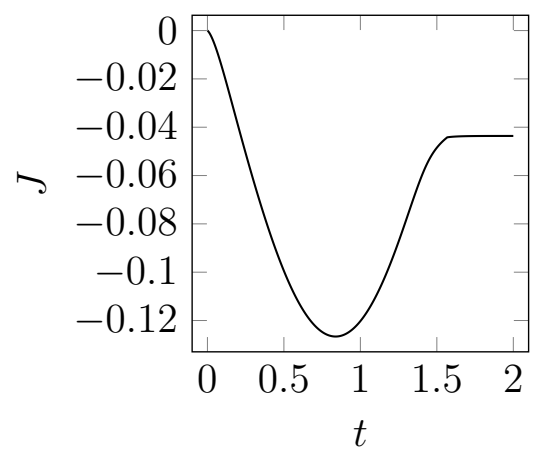

(c) $J(t)$.

Figure 4.7: The final filament configuration, bulk stress, $\sigma$, and $J(t)$ for the flexible filament $\left(\varepsilon=68.5\right.$ and $\left.V_{m}=1\right)$ solution in Figure 4.6. 


\section{Conclusion}

Understanding the origins of actomyosin contraction is an open problem in cellular biophysics, with implications for cell movement and division. In this paper, we presented a detailed investigation of how two a two-filament-motor system generates microscopic contraction if the filaments are flexible. We first derived a partial differential equation model, and described a method of computing in-plane stress. We then applied asymptotic analysis to a symmetric system with infinite free-moving motor velocity. The leading-order solution showed that two rigid filaments do not generate net stress if the motor traverses the entire length of the filaments. However, the introduction of filament bending enables the two-filament structure to generate net contraction. This is because bending breaks the polarity-reversal symmetry of rigid filaments. The resulting geometric asymmetry draws the plus-ends closer to parallel as the motor approaches. This facilitates faster motor movement when motors are close to filament plus-ends, and inhibits production of expansive stress.

Our analysis confirms that the microscopic dynamics of symmetric filament pairs and motors can explain contraction. We expect that the same mechanism also favours contraction in non-symmetric fibre-motor assemblies and that, consequently, macroscopic contraction in disordered networks could arise from the accumulation of multiple filament pairs, without the need for nonlinear amplification of contractile stress. Nevertheless, the question of how these results apply to networks, in which filaments cross at arbitrary angle and position, remains open. In Tam, Mogilner, and Oelz (2021) it has been confirmed that such networks relying on the biomechanical model for semi-flexible fibres and motors presented in this study do contract. We also mention that another potential approach is to derive a coarse-grained, continuum model based on the assumption of infinite filament length (Oelz 2014). This, and understanding how these microscopic mechanics give rise to structures including stress fibres (Pellegrin and Mellor 2007) and the contractile ring (Kamasaki, Osumi, and Mabuchi 2007; Svitkina 2018), will be subjects of future work.

\section{Acknowledgements}

The authors acknowledge funding from the Australian Research Council (ARC) Discovery Program (grant number DP180102956), awarded to D. B. O. and A. M.

\section{Declaration}

The authors have no conflicts of interest to declare. 


\section{References}

Belmonte, J. M., M. Leptin, and F. J. Nédélec (2017), "A theory that predicts behaviors of disordered cytoskeletal networks", Molecular Systems Biology 13, 941, DOI: 10.15252/ msb. 20177796.

Bidone, T. C., W. Jung, D. Maruri, C. Borau, R. D. Kamm, and T. Kim (2017), "Morphological transformation and force generation of active cytoskeletal networks", PLoS Computational Biology 13, e1005277, DOI: 10.1371/journal.pcbi.1005277.

Bormuth, V., V. Varga, J. Howard, and E. Schäffer (2009), "Protein friction limits diffusive and directed movements of kinesin motors in microtubules", Science 325, pp. 870-873, DOI: $10.1126 /$ science.1174923.

Broedersz, C. P. and F. C. Mackintosh (2014), "Modeling semiflexible polymer networks", Reviews of Modern Physics 86, pp. 995-1036, DOI: 10.1103/RevModPhys.86.995.

Chalut, K. J. and E. K. Paluch (2016), "The actin cortex: a bridge between cell shape and function", Developmental Cell 38, pp. 571-573, DOI: 10.1016/j.devce1.2016.09.011.

Cheffings, T. H., N. J. Burroughs, and M. K. Balasubramanian (2016), "Actomyosin ring formation and tension generation in eukaryotic cytokinesis", Current Biology 26, R719-R739, DOI: 10.1016/j.cub.2016.06.071.

Dasanayake, N. L., P. J. Michalski, and A. E. Carlsson (2011), "General mechanism of actomyosin contractility", Physical Review Letters 107, 118101, DOI: 10 .1103/ PhysRevLett.107.118101.

De La Cruz, E. M. and M. L. Gardel (2015), "Actin mechanics and fragmentation”, Journal of Biological Chemistry 290, pp. 17137-17144, DOI: 10.1074/jbc.R115.636472.

du Roure, O., A. Lindner, E. N. Nazockdast, and M. J. Shelley (2019), "Dynamics of flexible fibers in viscous flows and fluids", Annual Review of Fluid Mechanics 51, pp. 539-572, DOI: 10.1146/annurev-fluid-122316-045153.

Ennomani, H., G. Letort, C. Guérin, J. Martiel, W. Cao, F. J. Nédélec, E. M. De La Cruz, M. Théry, and L. Blanchoin (2016), "Architecture and connectivity govern actin network contractility", Current Biology 26, pp. 616-626, DOI: 10.1016/j.cub.2015.12.069.

Freedman, S. L., S. Banerjee, G. M. Hocky, and A. R. Dinner (2017), "A versatile framework for simulating the dynamic mechanical structure of cytoskeletal networks", Biophysical Journal 113, pp. 448-460, DOI: 10.1016/j.bpj.2017.06.003.

Freedman, S. L., G. M. Hocky, S. Banerjee, and A. R. Dinner (2018), "Nonequilibrium phase diagrams for actomyosin networks", Soft Matter 14, pp. 7740-7747, DOI: 10. 1039/c8sm00741a.

Gautel, M. (2011), "The sarcomeric cytoskeleton: who picks up the strain?", Current Opinion in Cell Biology 23, pp. 39-46, DOI: 10.1016/j.ceb.2010.12.001. 
Gittes, F., B. Mickey, J. Nettleton, and J. Howard (1993), "Flexural rigidity of microtubules and actin filaments measured from thermal fluctuations in shape", Journal of Cell Biology 120, pp. 923-934, DOI: 10.1083/jcb.120.4.923.

Head, D. A., A. J. Levine, and F. C. MacKintosh (2003), "Distinct regimes of elastic response and deformation modes of cross-linked cytoskeletal and semiflexible polymer networks", Physical Review E 68, 061907, DOI: 10.1103/PhysRevE.68.061907.

Hiraiwa, T. and G. Salbreux (2016), "Role of turnover in active stress generation in a filament network", Physical Review Letters 116, 188101, DOI: 10.1103/PhysRevLett. 116.188101.

Huxley, H. E. (2004), "Fifty years of muscle and the sliding filament hypothesis", European Journal of Biochemistry 271, pp. 1403-1415, DOI: 10.1111/j.1432-1033.2004.04044. $\mathrm{x}$.

Kamasaki, T., M. Osumi, and I. Mabuchi (2007), "Three-dimensional arrangement of F-actin in the contractile ring of fission yeast", Journal of Cell Biology 178, pp. 765-771, DOI: $10.1083 / \mathrm{jcb} .200612018$.

Kim, T. (2015), "Determinants of contractile forces generated in disorganized actomyosin bundles", Biomechanics and Modeling in Mechanobiology 14, pp. 345-355, DOI: 10. 1007/s10237-014-0608-2.

Komianos, J. E. and G. A. Papoian (2018), "Stochastic ratcheting on a funneled energy landscape is necessary for highly efficient contractility of actomyosin force diploes", Physical Review X 8, 021006, DOI: 10.1103/PhysRevX.8.021006.

Langer, J. and D. A. Singer (1984), "Knotted elastic curves in $\mathbb{R}^{3}$ ", Journal of the London Mathematical Society 30, pp. 512-520, DOI: 10.1112/j1ms/s2-30.3.512.

- (1987), "Curve-straightening in Riemannian manifolds", Annals of Global Analysis and Geometry 5, pp. 133-150, DOI: 10.1007/BF00127856.

Lenz, M. (2014), "Geometrical origins of contractility in disordered actomyosin networks", Physical Review X 4, 041002, DOI: 10.1103/PhysRevX.4.041002.

- (2020), "Reversal of contractility as a signature of self-organization in cytoskeletal bundles", eLife 9, e51751, DOI: 10.7554/eLife.51751.

Lenz, M., T. Thoresen, M. L. Gardel, and A. R. Dinner (2012), "Contractile units in disordered actomyosin bundles arise from F-actin buckling", Physical Review Letters 108, 238107, DOI: 10.1103/PhysRevLett.108.238107.

Letort, G., A. Z. Politi, H. Ennomani, M. Théry, F. J. Nédélec, and L. Blanchoin (2015), "Geometrical and mechanical properties control actin filament organization", PLoS Computational Biology 11, e1004245, DOI: 10.1371/journal.pcbi.1004245.

Linnér, A. (1989), "Some properties of the curve straightening flow in the plane", Transations of the Americal Mathematical Society 314, pp. 605-617, DOI: 10.1090/s00029947-1989-0989580-5. 
Linnér, A. (2003), "Symmetrized curve-straightening", Differential Geometry and its Applications 18, pp. 119-146, DOI: 10.1016/S0926-2245(02)00144-4.

McFadden, W. M., P. M. McCall, M. L. Gardel, and E. M. Munro (2017), "Filament turnover tunes both force generation and dissipation to control long-range flows in a model actomyosin cortex", PLoS Computational Biology 13, e1005811, DOI: 10.1371/ journal.pcbi.1005811.

Mogensen, P. K. and A. N. Risbeth (2018), "Optim: A mathematical optimization package for Julia", Journal of Open Source Software 3, 615, DOI: 10.21105/joss.00615.

Murrell, M. P. and M. L. Gardel (2012), "F-actin buckling coordinates contractility and severing in biomimetic actomyosin cortex", Proceedings of the National Academy of Science of the United States of America 109, pp. 20820-20825, DOI: 10.1073/pnas. 1214753109

Murrell, M. P., P. W. Oakes, M. Lenz, and M. L. Gardel (2015), "Forcing cells into shape: the mechanics of actomyosin contractility", Nature Reviews Molecular Cell Biology 16, pp. 486-498, DOI: $10.1038 / \mathrm{nrm} 4012$.

Oelz, D. B. (2011), "On the curve straightening flow of inextensible, open, planar curves", SeMA Journal: Bulletin of the Spanish Society of Applied Mathematics 54, pp. 5-24, DOI: $10.1007 / \mathrm{BF} 03322585$.

- (2014), "A viscous two-phase model for contractile actomyosin bundles", Journal of Mathematical Biology 68, pp. 1653-1676, DOI: 10.1007/s00285-013-0682-6.

Oelz, D. B., B. Y. Rubinstein, and A. Mogilner (2015), "A combination of actin treadmilling and cross-linking drives contraction of random actomyosin arrays", Biophysical Journal 109, pp. 1818-1829, DOI: 10.1016/j.bpj.2015.09.013.

Pellegrin, S. and H. Mellor (2007), "Actin stress fibres", Journal of Cell Science 120, pp. 3491-3499, DOI: 10.1242 /jcs. 018473.

Pollard, T. D. (2010), "Mechanics of cytokinesis in eukaryotes", Current Opinion in Cell Biology 22, pp. 50-56, DOI: 10.1016/j.ceb.2009.11.010.

Pollard, T. D. and B. O'Shaughnessy (2019), "Molecular mechanism of cytokinesis", Annual Review of Biochemistry 88, pp. 661-689, DOI: 10.1146/annurev-biochem062917012530.

Popov, K., J. E. Komianos, and G. A. Papoian (2016), "MEDYAN: Mechanochemical simulations of contraction and polarity alignment in actomyosin networks", PLoS Computational Biology 12, e1004877, DOI: 10.1371/journal.pcbi.1004877.

Reichl, E. M., Y. Ren, M. K. Morphew, M. Delannoy, J. C. Effler, K. D. Girard, S. Divi, P. A. Iglesias, S. C. Kuo, and D. N. Robinson (2008), "Interactions between myosin and actin crosslinkers control cytokinesis contractility dynamics and mechanics", Current Biology 18, pp. 471-480, DOI: 10.1016/j . cub.2008.02.056. 
Ronceray, P., C. P. Broedersz, and M. Lenz (2016), "Fiber networks amplify active stress", Proceedings of the National Academy of Science of the United States of America 113, pp. 2827-2832, DOI: 10.1073/pnas. 1514208113.

Soares e Silva, M., M. Depken, B. Stuhrmann, M. Korsten, F. C. MacKintosh, and G. H. Koenderink (2011), "Active multistage coarsening of actin networks driven by myosin motors", Proceedings of the National Academy of Science of the United States of America 108, pp. 9408-9413, DOI: 10.1073/pnas. 1016616108.

Stachowiak, M. R., C. Laplante, H. F. Chin, B. Guirao, E. Karatekin, T. D. Pollard, and B. O'Shaughnessy (2014), "Mechanism of cytokinetic contractile ring constriction in fission yeast", Developmental Cell 29, pp. 547-561, DOI: 10.1016/j.devce1.2014.04.021.

Svitkina, T. M. (2018), "Ultrastructure of the actin cytoskeleton", Current Opinion in Cell Biology 54, pp. 1-8, DOI: 10.1016/j.ceb.2018.02.007.

Tam, A. K. Y., A. Mogilner, and D. B. Oelz (2021), "Protein friction and filament bending facilitate contraction of disordered actomyosin networks", Biophysical Journal, in press, DOI: https://doi.org/10.1016/j.bpj.2021.08.012.

Tawada, K. and K. Sekimoto (1991), "Protein friction exerted by motor enzymes through a weak-binding interaction", Journal of Theoretical Biology 150, pp. 193-200, DOI: 10.1016/S0022-5193(05)80331-5.

Thoresen, T., M. Lenz, and M. L. Gardel (2011), "Reconstitution of contractile actomyosin bundles", Biophysical Journal 100, pp. 2698-2705, DOI: 10.1016/j . bpj .2011.04.031. Wen, Y. (1993), " $L^{2}$ flow of curve straightening in the plane", Duke Mathematics Journal 70, pp. 683-698, DoI: 10.1215/S0012-7094-93-07016-0.

- (1995), "Curve straightening flow deforms closed plane curves with nonzero rotation number to circles", Journal of Differential Equations 120, pp. 89-107, DOI: 10.1006/ jdeq.1995.1106.

Yamada, K. M. and M. Sixt (2019), "Mechanisms of 3D cell migration", Nature Reviews Molecular Cell Biology 20, pp. 738-752, DOI: 10.1038/s41580-019-0172-9.

Yu, Q., J. Li, M. P. Murrell, and T. Kim (2018), "Balance between force generation and relaxation leads to pulsed contraction of actomyosin networks", Biophysical Journal 115, pp. 2003-2013, DOI: 10.1016/j.bpj.2018.10.008. 


\section{A Mathematical Model Derivation}

In this Appendix, we present a detailed derivation of the PDE model (2.14)-(2.16). We derive the system of force-balance PDEs using the variational principle. The energy functional for the structure consisting of a myosin motor attached to two semi-flexible actin filaments is

$$
\begin{gathered}
E\left[z_{1}, z_{2}, m_{1}, m_{2}\right]:=\sum_{i=1}^{2} \int_{0}^{L_{i}}\left(\frac{\lambda_{a}}{2 \Delta t}\left|z_{i}-\boldsymbol{F} z_{i}^{n}\right|^{2}+\frac{\kappa_{a}}{2}\left|z_{i}^{\prime \prime}\right|^{2}+\frac{1}{\delta_{a}}\left(\left|z_{i}^{\prime}\right|-1\right)^{2}\right) \mathrm{d} s \\
+\frac{1}{\delta_{m}}\left|z_{1}\left(m_{1}, t\right)-z_{2}\left(m_{2}, t\right)\right|^{2}+\sum_{j=1}^{2}\left(-F_{s} m_{j}+\frac{F_{s}}{V_{m}} \frac{\left(m_{j}-m_{j}^{n}\right)^{2}}{2 \Delta t}\right),
\end{gathered}
$$

where $z_{i}(s, t)=\left(x_{i}(s, t), y_{i}(s, t)\right)$, for $i=1,2$, are the filament shapes and positions, $m_{1}(t)$, and $m_{2}(t)$ are the motor relative positions, and

$$
\boldsymbol{F}=\left[\begin{array}{ll}
L_{x x} / L_{x x}^{n} & L_{y x} / L_{y y}^{n} \\
L_{x y} / L_{x x}^{n} & L_{y y} / L_{y y}^{n}
\end{array}\right]
$$

is the deformation gradient tensor. According to the variational principle underlying the derivation, the solution at the next time step is such that the functional derivative with respect to all degrees of freedom vanishes, that is

$$
\frac{\delta E}{\delta z_{1}}=0, \quad \frac{\delta E}{\delta z_{2}}=0, \quad \frac{\delta E}{\delta m_{1}}=0, \quad \text { and } \quad \frac{\delta E}{\delta m_{2}}=0
$$

Evaluating each of (A.3) gives rise to the variational equations

$$
\left\{\begin{aligned}
0= & \int_{0}^{L_{1}} \frac{\lambda_{a}}{\Delta t} \delta z_{1} \cdot\left(z_{1}-\boldsymbol{F} z_{1}^{n}\right)+\kappa_{a} z_{1}^{\prime \prime} \cdot \delta z_{1}^{\prime \prime}+ \\
& +\frac{2}{\delta_{a}}\left(\left|z_{1}^{\prime}\right|-1\right) \frac{z_{1}^{\prime} \cdot \delta z_{1}^{\prime}}{\left|z_{1}^{\prime}\right|} \mathrm{d} s+\frac{2}{\delta_{m}}\left(z_{1}\left(m_{1}, t\right)-z_{2}\left(m_{1}, t\right)\right) \cdot \delta z_{1}\left(m_{1}, t\right) \\
0= & \int_{0}^{L_{2}} \frac{\lambda_{a}}{\Delta t} \delta z_{2} \cdot\left(z_{2}-\boldsymbol{F} z_{2}^{n}\right)+\kappa_{a} z_{2}^{\prime \prime} \cdot \delta z_{2}^{\prime \prime}+ \\
& +\frac{2}{\delta_{a}}\left(\left|z_{2}^{\prime}\right|-1\right) \frac{z_{2}^{\prime} \cdot \delta z_{2}^{\prime}}{\left|z_{2}^{\prime}\right|} \mathrm{d} s-\frac{2}{\delta_{m}}\left(z_{1}\left(m_{1}, t\right)-z_{2}\left(m_{1}, t\right)\right) \cdot \delta z_{2}\left(m_{2}, t\right), \\
0= & \delta m_{1}\left[-F_{s}+\frac{F_{s}}{V_{m}} \frac{\left(m_{1}-m_{1}^{n}\right)}{\Delta t}+\frac{2}{\delta_{m}}\left(z_{1}\left(m_{1}, t\right)-z_{2}\left(m_{1}, t\right)\right) \cdot z_{1}^{\prime}\left(m_{1}, t\right)\right] \\
0= & \delta m_{2}\left[-F_{s}+\frac{F_{s}}{V_{m}} \frac{\left(m_{2}-m_{2}^{n}\right)}{\Delta t}-\frac{2}{\delta_{m}}\left(z_{1}\left(m_{1}, t\right)-z_{2}\left(m_{1}, t\right)\right) \cdot z_{2}^{\prime}\left(m_{2}, t\right)\right] .
\end{aligned}\right.
$$

For the motor evolution equations (A.4e) and (A.4f), we can immediately apply the continuum limit $\Delta t \rightarrow 0$, for which $\dot{m}_{i}=\left(m_{i}-m_{i}^{n}\right) / \Delta t$ for $i=1,2$. In addition, we consider the limit $\delta_{m} \rightarrow 0$ and replace the forces due to motor stretching by the force $\mu$ 
which represents the Lagrange multiplier for the constraint

$$
z\left(m_{1}, t\right)=z\left(m_{2}, t\right)
$$

This yields the ordinary differential equations

$$
\begin{aligned}
& \frac{\mathrm{d} m_{1}}{\mathrm{~d} t}=V_{m}\left[1-\frac{\mu}{F_{s}} \frac{z_{1}-z_{2}}{\left\|z_{1}-z_{2}\right\|} \cdot z_{1}^{\prime}\left(m_{1}, t\right)\right], \\
& \frac{\mathrm{d} m_{2}}{\mathrm{~d} t}=V_{m}\left[1+\frac{\mu}{F_{s}} \frac{z_{1}-z_{2}}{\left\|z_{1}-z_{2}\right\|} \cdot z_{2}^{\prime}\left(m_{2}, t\right)\right],
\end{aligned}
$$

which are force-balance equations for the myosin motor positions $m_{1}(t)$ and $m_{2}(t)$. The equations (A.6) represent that unloaded motors (for which $\mu=0$ ) move at the free-moving velocity, $V_{m}$. As the motor moves, it is exposed to stretching forces, with magnitude given by the Lagrange multiplier $\mu$. The term involving the dot product is the projection of this force onto the direction of motor movement along the $i$-th filament. Assuming a linear force-velocity relationship its ratio with the stall force, $F_{s}$, determines the reduction of motor speed due to stretching forces.

For the filament equations (A.4b) and (A.4d), in addition to $\delta_{m} \rightarrow 0$, we also let $\delta_{a} \rightarrow 0$ enforcing the constraints

$$
\left|z_{i}^{\prime}\right|=1, \quad i=1,2 .
$$

We write the limits of $2\left(\left|z_{i}^{\prime}\right|-1\right) / \delta_{a}$ as $\lambda_{i}$ and apply integration by parts to remove derivatives of $\delta z_{i}$ from under the integral sign. This yields

$$
\begin{aligned}
\int \kappa_{a} z_{i}^{\prime \prime} \cdot \delta z_{i}^{\prime \prime}+\lambda_{i} z_{i}^{\prime} \cdot \delta z_{i}^{\prime} \mathrm{d} s & =\int \delta z_{i} \cdot\left[\kappa_{a} z_{i}^{\prime \prime \prime \prime}-\left(\lambda_{i} z_{i}^{\prime}\right)^{\prime}\right] \mathrm{d} s \\
& +\delta z_{i} \cdot\left(\lambda_{i} z_{i}^{\prime}-\kappa_{a} z_{i}^{\prime \prime \prime}\right)+\kappa_{a} \delta z_{1}^{\prime} \cdot z_{i}^{\prime \prime} .
\end{aligned}
$$

We then rewrite equations (A.4b) and (A.4d) as

$$
\begin{gathered}
\int_{0}^{L_{1}} \delta z_{1} \cdot\left[\frac{\lambda_{a}}{\Delta t}\left(z_{1}-\boldsymbol{F} z_{1}^{n}\right)+\kappa_{a} z_{1}^{\prime \prime \prime \prime}-\left(\lambda_{1} z_{1}^{\prime}\right)^{\prime}+\mu \frac{z_{1}-z_{2}}{\left\|z_{1}-z_{2}\right\|} \delta\left(s-m_{1}\right)\right] \mathrm{d} s \\
+\left[\delta z_{1} \cdot\left(\lambda_{1} z_{1}^{\prime}-\kappa_{a} z_{1}^{\prime \prime \prime}\right)+\kappa_{a} \delta z_{1}^{\prime} \cdot z_{1}^{\prime \prime}\right]_{0}^{L_{1}}, \\
\int_{0}^{L_{2}} \delta z_{2} \cdot\left[\frac{\lambda_{a}}{\Delta t}\left(z_{2}-\boldsymbol{F} z_{2}^{n}\right)+\kappa_{a} z_{2}^{\prime \prime \prime \prime}-\left(\lambda_{2} z_{2}^{\prime}\right)^{\prime}-\mu \frac{z_{1}-z_{2}}{\left\|z_{1}-z_{2}\right\|} \delta\left(s-m_{2}\right)\right] \mathrm{d} s \\
+\left[\delta z_{2} \cdot\left(\lambda_{2} z_{2}^{\prime}-\kappa_{a} z_{2}^{\prime \prime \prime}\right)+\kappa_{a} \delta z_{2}^{\prime} \cdot z_{2}^{\prime \prime}\right]_{0}^{L_{2}}
\end{gathered}
$$

where $\delta$ is the Dirac delta function. The equations (A.9) enable us to derive the continuum governing equations and boundary conditions. First, we require the filaments to have zero curvature at their tips,

$$
z_{i}^{\prime \prime}=0 \quad \text { at } \quad s=0, L_{i}
$$


The remaining boundary terms in (A.9) then give rise to the conditions

$$
\lambda_{i} z_{i}^{\prime}-\kappa_{a} z_{i}^{\prime \prime \prime}=0 \quad \text { at } \quad s=0, L_{i}
$$

which specifies that the boundary values vanish at $s=0, L_{i}$. Next, we apply the fundamental lemma of the calculus of variations to the remaining integrals. In the continuum limit $\Delta t \rightarrow 0$ for which $\boldsymbol{F}=\boldsymbol{I}$, and $\left(z_{i}-z_{i}^{n}\right) / \Delta t=\dot{z}_{i}$, we obtain

$$
\begin{array}{lll}
\lambda_{a} \frac{\partial z_{1}}{\partial t}+\kappa_{a} z_{1}^{\prime \prime \prime \prime}-\left(\lambda_{1} z_{1}^{\prime}\right)^{\prime}+\mu \frac{z_{1}-z_{2}}{\left\|z_{1}-z_{2}\right\|} \delta\left(s-m_{1}\right)=0 & \text { on } \quad\left(0<s<L_{1}\right), \\
\lambda_{a} \frac{\partial z_{2}}{\partial t}+\kappa_{a} z_{2}^{\prime \prime \prime \prime}-\left(\lambda_{2} z_{2}^{\prime}\right)^{\prime}-\mu \frac{z_{1}-z_{2}}{\left\|z_{1}-z_{2}\right\|} \delta\left(s-m_{2}\right)=0 & \text { on } \quad\left(0<s<L_{2}\right) .
\end{array}
$$

We commence the analysis by considering the ansatz for rigid filaments. Taking the time derivative of (3.3) gives $\partial_{t} z_{0}^{\prime}=-z_{0}^{\prime \perp} \dot{\theta} / 2$, and taking four spatial derivatives of (3.7) yields $z_{1}^{(5)}=h^{(5)} z_{0}^{\prime \perp}$. Next, we can differentiate the governing equation of $\mathcal{O}(1)$ (3.6a) once with respect to $s$, and substitute the two above expressions to obtain

$$
-z_{0}^{\prime \perp} \frac{\dot{\theta}}{2}+h^{(5)} z_{0}^{\prime \perp}-\lambda_{0}^{\prime \prime} z_{0}^{\prime}+\mu_{0}\left(\begin{array}{l}
1 \\
0
\end{array}\right) \delta^{\prime}\left(s-m_{0}\right)=0 .
$$

We can now multiply the boundary condition (3.6c) by $z_{0}^{\prime}$, and use the property $z_{1}^{\prime \prime \prime} \cdot z_{0}^{\prime}=0$, which follows from (3.6e), to obtain $\lambda_{0}(0)=\lambda_{0}(1)=0$. Finally, multiplying (B.1) by $z_{0}^{\prime}$ implies that

$$
\lambda_{0}=\mu_{0}\left(\begin{array}{l}
1 \\
0
\end{array}\right) \cdot z_{0}^{\prime}\left(H\left(s-m_{0}\right)-s\right)=H\left(s-m_{0}\right)-s,
$$


where $H(s)$ denotes the Heaviside step function. The Lagrange multiplier $\mu_{0}=1 / \sin (\theta / 2)$, by rearranging (3.6b). The leading-order bulk stress is then

$$
\sigma_{0}=-2 \int_{0}^{1} \lambda_{0} \mathrm{~d} s=-2 \mu_{0} \sin \left(\frac{\theta}{2}\right)\left(\frac{1}{2}-m_{0}\right)=-2\left(\frac{1}{2}-m_{0}\right)=2 \nu_{0}
$$

where $\nu_{0}=m_{0}-1 / 2$. This completes the derivation of the quantities listed in (3.8).

We now derive the ordinary differential equations (3.9) for $y_{0}, \theta$, and $\nu_{0}$, the three degrees of freedom that govern the leading-order filament position, $z_{0}$. On taking the time derivative and variation, the ansatz (3.5) implies that

$$
\begin{aligned}
& \partial_{t} z_{0}=\left(\begin{array}{c}
0 \\
\dot{y}_{0}
\end{array}\right)-z_{0}^{\prime} \dot{m}_{0}-z_{0}^{\prime \perp}\left(s-m_{0}\right) \frac{\dot{\theta}}{2} \\
& \delta z_{0}=\left(\begin{array}{c}
0 \\
\delta y_{0}
\end{array}\right)-z_{0}^{\prime} \delta m_{0}-z_{0}^{\prime \perp}\left(s-m_{0}\right) \frac{\delta \theta}{2}
\end{aligned}
$$

Integrating (3.6a) against $\delta z_{0}$ then yields

$$
\begin{aligned}
0 & =\int_{0}^{1} \partial_{t} z_{0} \cdot \delta z_{0} \mathrm{~d} s+\mu_{0}\left(\begin{array}{l}
1 \\
0
\end{array}\right) \cdot\left(\left(\begin{array}{c}
0 \\
\delta y_{0}
\end{array}\right)-z_{0}^{\prime} \delta m_{0}\right) \\
& =\int_{0}^{1} \partial_{t} z_{0} \cdot \delta z_{0} \mathrm{~d} s-\mu_{0} \sin (\theta / 2) \delta m_{0} \\
& =\int_{0}^{1} \partial_{t} z_{0} \cdot \delta z_{0} \mathrm{~d} s-\delta m_{0} .
\end{aligned}
$$

Substituting (B.4) into (B.5) then gives

$$
\begin{aligned}
0 & =\int_{0}^{1}\left(\left(\begin{array}{c}
0 \\
\dot{y}_{0}
\end{array}\right)-z_{0}^{\prime} \dot{m}_{0}-z_{0}^{\perp \perp}\left(s-m_{0}\right) \frac{\dot{\theta}}{2}\right) \cdot\left(\left(\begin{array}{c}
0 \\
\delta y_{0}
\end{array}\right)-z_{0}^{\prime} \delta m_{0}-z_{0}^{\prime \perp}\left(s-m_{0}\right) \frac{\delta \theta}{2}\right) \mathrm{d} s-\delta m_{0} \\
& =\left(\begin{array}{c}
0 \\
\delta y_{0}
\end{array}\right) \cdot \int_{0}^{1}\left(\left(\begin{array}{c}
0 \\
\dot{y}_{0}
\end{array}\right)-z_{0}^{\prime} \dot{m}_{0}-z_{0}^{\prime \perp}\left(s-m_{0}\right) \frac{\dot{\theta}}{2}\right) \mathrm{d} s-\delta m_{0}\left(\left(\begin{array}{c}
0 \\
\dot{y}_{0}
\end{array}\right) \cdot z_{0}^{\prime}-\dot{m}_{0}\right) \\
& -\int_{0}^{1}\left(z_{0}^{\prime \perp} \cdot\left(\begin{array}{c}
0 \\
\dot{y}_{0}
\end{array}\right)-\left(s-m_{0}\right) \frac{\dot{\theta}}{2}\right) \cdot\left(s-m_{0}\right) \frac{\delta \theta}{2} \mathrm{~d} s-\delta m_{0} \\
& =\delta y_{0} \int_{0}^{1}\left(\dot{y}_{0}-\cos (\theta / 2) \dot{m}_{0}-\sin (\theta / 2)\left(s-m_{0}\right) \frac{\dot{\theta}}{2}\right) \mathrm{d} s-\delta m_{0}\left(\dot{y}_{0} \cos (\theta / 2)-\dot{m}_{0}\right) \\
& -\int_{0}^{1}\left(\dot{y}_{0} \sin (\theta / 2)-\left(s-m_{0}\right) \frac{\dot{\theta}}{2}\right) \cdot\left(s-m_{0}\right) \frac{\delta \theta}{2} \mathrm{~d} s-\delta m_{0} \\
& =\delta y_{0}\left(\dot{y}_{0}-\cos (\theta / 2) \dot{m}_{0}-\sin (\theta / 2)\left(1 / 2-m_{0}\right) \frac{\dot{\theta}}{2}\right)-\delta m_{0}\left(1+\dot{y}_{0} \cos (\theta / 2)-\dot{m}_{0}\right) \\
& -\left(\dot{y}_{0} \sin (\theta / 2)\left(1 / 2-m_{0}\right)-\left(1 / 3-m_{0}+m_{0}^{2}\right) \frac{\dot{\theta}}{2}\right) \frac{\delta \theta}{2} .
\end{aligned}
$$


Collecting the coefficients of $\delta y_{0}, \delta \theta$ and $\delta m_{0}$, we obtain the system of differential equations (writing $\nu_{0}=m_{0}-1 / 2$ )

$$
\begin{aligned}
\dot{y}_{0} & =\left(12 \nu_{0}^{2}+1\right) \cot \left(\frac{\theta}{2}\right) \csc \left(\frac{\theta}{2}\right), \\
\dot{\theta} & =-24 \nu_{0} \cot \left(\frac{\theta}{2}\right), \\
\dot{\nu}_{0} & =\csc ^{2}\left(\frac{\theta}{2}\right)\left(1+6 \nu_{0}^{2}(\cos (\theta)+1)\right),
\end{aligned}
$$

where $\csc (\phi)=1 / \sin (\phi)$. The equations (B.6b) and (B.6c) for $\theta$ and $\nu_{0}$ are also independent of $y_{0}$, suggesting that the solution is invariant to vertical translations. Furthermore, the trigonometric functions can be eliminated by writing $S=\sin ^{2}(\theta / 2)$, which yields

$$
\begin{gathered}
\frac{\mathrm{d} S}{\mathrm{~d} t}=-24 \nu_{0}(1-S), \\
S \frac{\mathrm{d} \nu_{0}}{\mathrm{~d} t}=1+12 \nu_{0}^{2}(1-S) .
\end{gathered}
$$

This completes the derivation of equation (3.9).

\section{B.2 Higher-Order Corrections}

To obtain the higher-order corrections, we first use the leading-order equation (B.1) and the ansatz (3.7) to solve for $h$, the curvature of $z_{1}$. Multiplying (B.1) by $z_{0}^{\perp \perp}$ and using $z_{1}^{\prime \prime \prime} \cdot z_{0}^{\prime}=0$ (which follows from $(3.6 \mathrm{e})$ ), we obtain $h^{\prime \prime \prime}(0)=h^{\prime \prime \prime}(1)=0$, and subsequently

$$
-\frac{\dot{\theta}}{2}+h^{\prime \prime \prime \prime \prime \prime}-\mu_{0} \cos \left(\frac{\vartheta}{2}\right) \delta^{\prime}\left(s-m_{0}\right)=0
$$

The boundary conditions $h^{\prime \prime \prime}(0)=0=h^{\prime \prime \prime}(1)$ imply that

$$
h^{\prime \prime \prime}=\frac{\dot{\vartheta}}{2} \frac{s(s-1)}{2}+\mu_{0} \cos \left(\frac{\vartheta}{2}\right)\left(H\left(s-m_{0}\right)-s\right)
$$

and furthermore (since $\left.z_{1}^{\prime \prime}(0)=0=z_{1}^{\prime \prime}(1)\right)$, substituting for $\dot{\theta}$ and $\mu_{0}$,

$$
h^{\prime \prime}=-\cot \left(\frac{\theta}{2}\right)\left[\left(m_{0}-s\right) H\left(s-m_{0}\right)+s^{2}\left(m_{0}(2 s-3)-s+2\right)\right],
$$

which is an expression for filament curvature, $h^{\prime \prime}(s, t)$.

We now obtain the perturbation solution for the bulk stress, $\sigma_{1}$, which requires knowledge of the quantities $\mu_{1}$ and $\lambda_{1}$. First, we integrate (B.10) once with respect to $s$. This introduces another constant of integration, here denoted $A(t)$, which might be 
time-dependent and cannot be determined from boundary data. Consequently, we write

$$
h^{\prime}(s, t)=\tilde{h}^{\prime}(s, t)+A(t)
$$

where

$$
\tilde{h}^{\prime}(s, t)=\frac{\dot{\theta}}{2} \frac{s^{3}}{12}\left(\frac{s}{2}-1\right)+\mu_{0} \cos \left(\frac{\theta}{2}\right)\left(\frac{1}{2}(m-s)^{2} H(s-m)-\frac{s^{3}}{6}\right) .
$$

Collecting the coefficients of $\varepsilon$ in the governing equations with asymptotic expansions, we obtain the $\mathcal{O}(\varepsilon)$ problem (3.14). On taking a derivative of (3.14a) and substituting $z_{1}^{\prime}=h^{\prime}(s, t) z_{0}^{\prime \perp}=\left(A(t)+\tilde{h}^{\prime}(s, t)\right) z_{0}^{\prime \perp}$, we obtain

$$
\begin{gathered}
\left(\dot{A}+\partial_{t} \tilde{h}^{\prime}\right) z_{0}^{\prime \perp}+z_{2}^{\prime \prime \prime \prime \prime}-\left(\left(\lambda_{0}\left(A+f^{\prime}(t, s)\right)\right)^{\prime \prime} z_{0}^{\prime \perp}+\lambda_{1}^{\prime \prime} z_{0}^{\prime}\right) \\
+\mu_{1}\left(\begin{array}{l}
1 \\
0
\end{array}\right) \delta^{\prime}\left(s-m_{0}\right)-\mu_{0}\left(\begin{array}{l}
1 \\
0
\end{array}\right) \delta^{\prime \prime}\left(s-m_{0}\right) m_{1}=0
\end{gathered}
$$

Expanding the inextensibility constraint (3.14f) implies that $z_{0}^{\prime} \cdot z_{2}^{(5)}=-\left(\left|z_{1}^{\prime}\right|^{2}\right)^{(4)} / 2$. Multiplying (B.13) by $z_{0}^{\prime}$, we obtain

$$
-\frac{1}{2}\left(\left|z_{1}^{\prime}\right|^{2}\right)^{(4)}-\lambda_{1}^{\prime \prime}+\left(\begin{array}{l}
1 \\
0
\end{array}\right) \cdot z_{0}^{\prime}\left[\mu_{1} \delta^{\prime}\left(s-m_{0}\right)-\mu_{0} \delta^{\prime \prime}\left(s-m_{0}\right) m_{1}\right]=0 .
$$

We can integrate (B.14) twice and apply the boundary conditions (3.14c) to determine the constants of integration. This yields

$$
\frac{1}{2}\left(\left|z_{1}^{\prime}\right|^{2}\right)^{\prime \prime}+\lambda_{1}=\left(\begin{array}{l}
1 \\
0
\end{array}\right) \cdot z_{0}^{\prime}\left[\mu_{1}\left(H\left(s-m_{0}\right)-\frac{s}{L}\right)-\mu_{0} \delta\left(s-m_{0}\right) m_{1}\right]
$$

which we can rearrange to obtain $\lambda_{1}$. To eliminate $\mu_{1}$ from (B.15), we use (3.14b) to infer an expression for $\mu_{1}$. Substituting the ansatzes (3.5) and (3.7) for $z_{0}$ and $z_{1}$ respectively, we obtain

$$
\begin{aligned}
0 & =-\left(\begin{array}{l}
1 \\
0
\end{array}\right) \cdot\left(\mu_{1} z_{0}^{\prime}+\mu_{0} h^{\prime}(s, t) z_{0}^{\prime \perp}\left(m_{0}, t\right)\right) \\
& =-\left(\sin \left(\frac{\theta}{2}\right) \mu_{1}-\cos \left(\frac{\theta}{2}\right) \mu_{0} h^{\prime}\left(m_{0}, t\right)\right),
\end{aligned}
$$

and therefore

$$
\mu_{1}=\mu_{0} h^{\prime}\left(m_{0}, t\right) \frac{\cos (\theta / 2)}{\sin (\theta / 2)}=\mu_{0}\left(A+\tilde{h}^{\prime}\left(m_{0}, t\right)\right) \cot \left(\frac{\theta}{2}\right)
$$


Substituting (B.16) into (B.15), we obtain

$$
\begin{aligned}
\lambda_{1} & =\left(\begin{array}{l}
1 \\
0
\end{array}\right) \cdot z_{0}^{\prime}\left[\left(\mu_{0}\left(A+\tilde{h}^{\prime}\left(m_{0}, t\right)\right) \cot \left(\frac{\theta}{2}\right)\right)\left(H\left(s-m_{0}\right)-s\right)-\mu_{0} \delta\left(s-m_{0}\right) m_{1}\right] \\
& -\frac{1}{2}\left(\left|z_{1}^{\prime}\right|^{2}\right)^{\prime \prime} \\
& =\left(A+\tilde{h}^{\prime}\left(m_{0}, t\right)\right) \cot \left(\frac{\theta}{2}\right)\left(H\left(s-m_{0}\right)-s\right)-\delta\left(s-m_{0}\right) m_{1}-\left(z_{1}^{\prime} \cdot z_{1}^{\prime \prime}\right)^{\prime}
\end{aligned}
$$

Using the simplified expression (B.17) for $\lambda_{1}$, we can write the first-order perturbation of the bulk stress (3.12), given by

$$
\begin{aligned}
\sigma_{1} & =-2 \int_{0}^{1}\left(\left|z_{1}^{\prime \prime}\right|^{2}+\lambda_{1}\right) \mathrm{d} s \\
& =-2 \int_{0}^{1}\left|h^{\prime \prime}\right|^{2} \mathrm{~d} s-2\left(A+\tilde{h}^{\prime}\left(m_{0}, t\right)\right) \cot \left(\frac{\theta}{2}\right)\left(\frac{1}{2}-m_{0}\right)+2 m_{1}
\end{aligned}
$$

where $\tilde{h}^{\prime}\left(m_{0}, t\right)$ is

$$
\begin{aligned}
\tilde{h}^{\prime}\left(m_{0}, t\right) & =\frac{\dot{\theta}}{2} \frac{m_{0}^{3}}{12}\left(\frac{m_{0}}{2}-1\right)+\cot \left(\frac{\theta}{2}\right)\left(-\frac{m_{0}^{3}}{6}\right) \\
& =\frac{1}{2}\left(-12\left(2 m_{0}-1\right) \cot \left(\frac{\theta}{2}\right)\right) \frac{m_{0}^{3}}{12}\left(\frac{m_{0}}{2}-1\right)+\cot \left(\frac{\theta}{2}\right)\left(-\frac{m_{0}^{3}}{6}\right) \\
& =\cot \left(\frac{\theta}{2}\right)\left(-\frac{m_{0}^{3}}{12}\right)\left[\left(12 m_{0}-6\right)\left(\frac{m_{0}}{2}-1\right)+2\right] \\
& =-\frac{1}{12} m_{0}^{3}\left(6 m_{0}^{2}-15 m_{0}+8\right) \cot \left(\frac{\theta}{2}\right) .
\end{aligned}
$$

To solve for the first-order correction to the filament positions, $z_{1}$, we require an initial condition, here denoted $z_{I, 1}(s)=z_{1}(s, 0)$. To determine the asymptotic expansion of the initial condition (2.28c), we return to the force-balance equations (2.1), and its equivalent time-discrete energy minimisation problem for the functional (2.2). In this expression, the drag component (2.3) dominates when $\Delta t$ is small. Therefore, we determine the leading order term in the asymptotic expansion of the initial condition $z_{I}=z_{I, 0}+\varepsilon z_{I, 0}+\ldots$ as the best approximation of $z_{I}$ in $L^{2}$ among the straight fibres (3.5), that is

$$
z_{I, 0}=\left(\begin{array}{c}
0 \\
y_{I, 0}
\end{array}\right)+\left(\begin{array}{c}
\sin \left(\theta_{I} / 2\right) \\
\cos \left(\theta_{I} / 2\right)
\end{array}\right)\left(s-m_{I, 0}\right)
$$

where

$$
\left(m_{I, 0}, y_{I, 0}, \theta_{I}\right)=\operatorname{argmin}_{\bar{m}, \bar{y}, \bar{\theta}} \int_{0}^{1}\left(z_{I, 0}-z_{I}\right)^{2} \mathrm{~d} s .
$$

Since we focus on pairs of initially straight fibres in this study, we set $z_{I}=z_{I, 0}$. 
A similar approach is available to determine $z_{1}^{I}$. Using the ansatz (3.7), we have

$$
z_{I, 1}=\left(\begin{array}{c}
0 \\
y_{I, 1}(t)
\end{array}\right)-z_{I, 0}^{\prime}(t) m_{I, 1}+z_{I, 0}^{\perp}\left(A_{I}\left(s-m_{I, 0}\right)+\int_{m_{I, 0}}^{s} \tilde{h}^{\prime}(t, s) \mathrm{d} s\right)
$$

where

$$
\left(m_{I, 1}, y_{I, 1}, A_{I}\right)=\operatorname{argmin}_{\bar{m}, \bar{y}, \bar{\theta}} \int_{0}^{1}\left(z_{I, 0}+\varepsilon z_{I, 1}-z_{I}\right)^{2} \mathrm{~d} s .
$$

In the case where $z_{I, 0}=z_{I}$ the term $z_{I, 1}$ is minimal in $L^{2}$. Then, the degrees of freedom $m_{I, 1}, y_{I, 1}$, and $A_{I}$ can be computed using

$$
0=\int_{0}^{1} z_{I, 1} \cdot \delta z_{I, 1} \mathrm{~d} s, \quad \text { where } \quad \delta z_{I, 1}=\left(\begin{array}{c}
0 \\
\delta y_{1}
\end{array}\right)-z_{I, 0}^{\prime} \delta m_{1}+z_{I, 0}^{\prime \perp}\left(s-m_{I, 0}\right) \delta A
$$

When we set $\delta y_{1}=y_{0}, \delta m_{1}=\int_{0}^{1}\left(m_{0}-s\right) \mathrm{d} s$, and $\delta A=0$, we find that

$$
0=\int_{0}^{1} z_{I, 1} \cdot\left[\left(\begin{array}{c}
0 \\
y_{I, 0}
\end{array}\right)+z_{I, 0}^{\prime}\left(s-m_{I, 0}\right)\right]=\int_{0}^{1} z_{I, 1} \cdot z_{I, 0} \mathrm{~d} s .
$$

It therefore holds that $J_{1}(0)=0$.

To complete the derivation, we use the ansatz (3.7) with degrees of freedom $A(t), y_{1}(t)$, and $m_{1}(t)$. Its variation and time-derivative are given by

$$
\begin{aligned}
\delta z_{1} & =\left(\begin{array}{c}
0 \\
\delta y_{1}
\end{array}\right)-z_{0}^{\prime} \delta m_{1}+z_{0}^{\prime \perp}\left(s-m_{0}\right) \delta A \\
\partial_{t} z_{1} & =\left(\begin{array}{c}
0 \\
\dot{y}_{1}(t)
\end{array}\right)+z_{0}^{\prime}\left[-\dot{m}_{1}+\frac{\dot{\theta}}{2}\left(A(t)\left(s-m_{0}\right)+\int_{m_{0}}^{s} \tilde{h}^{\prime}(s, t) \mathrm{d} s\right)\right] \\
& +z_{0}^{\prime \perp}\left(\frac{\dot{\theta}}{2} m_{1}+\dot{A}(t)\left(s-m_{0}\right)-A \dot{m}_{0}+\int_{m_{0}}^{s} \partial_{t} \tilde{h}^{\prime}(s, t) \mathrm{d} s-\tilde{h}^{\prime}\left(m_{0}, t\right) \dot{m}_{0}\right)
\end{aligned}
$$

where $\tilde{h}^{\prime}\left(m_{0}, t\right)$ is given in (B.19). A system of differential equations for $m_{1}, A$ and $y_{1}$ can then be found integrating (3.14a) against $\delta z_{1}$. Using computer algebra, we obtained the 
system

$$
\begin{aligned}
\frac{\mathrm{d} y_{1}}{\mathrm{~d} t} & =\frac{1}{960} \csc ^{4}\left(\frac{\theta}{2}\right)\left(960 A \sin \left(\frac{\theta}{2}\right)\left(6 \nu_{0}^{2} \cos (\theta)+6 \nu_{0}^{2}+1\right)\right. \\
& +\cos \left(\frac{\theta}{2}\right)\left(-8928 \nu_{0}^{7}+9600 \nu_{0}^{5}-720 \nu_{0}^{4}+490 \nu_{0}^{3}+2940 \nu_{0}^{2}+3 \nu_{0}\left(960 m_{1}-17\right)+240\right) \\
& \left.-3 \cos \left(\frac{3 \theta}{2}\right)\left(864 \nu_{0}^{7}-960 \nu_{0}^{5}-240 \nu_{0}^{4}+30 \nu_{0}^{3}+980 \nu_{0}^{2}+3 \nu_{0}+960 \nu_{0} m_{1}+80\right)\right), \\
\frac{\mathrm{d} m_{1}}{\mathrm{~d} t} & =-\frac{1}{960} \csc ^{4}\left(\frac{\theta}{2}\right)\left(-5760 A \nu_{0}^{2} \sin (\theta)-480 A \sin (\theta)\right. \\
& +10\left(576 \nu_{0}^{7}-624 \nu_{0}^{5}-20 \nu_{0}^{3}+3 \nu_{0}+48\right) \cos (\theta)+4608 \nu_{0}^{7}-5040 \nu_{0}^{5}-200 \nu_{0}^{3} \\
& \left.-1440 \nu_{0}^{2}+21 \nu_{0}+3 \nu_{0} \cos (2 \theta)\left(384 \nu_{0}^{6}-400 \nu_{0}^{4}+480 \nu_{0}+480 m_{1}+3\right)-1440 \nu_{0} m_{1}-480\right) \\
\frac{\mathrm{d} A}{\mathrm{~d} t} & =-\frac{1}{160} \cot \left(\frac{\theta}{2}\right) \csc ^{2}\left(\frac{\theta}{2}\right)\left(4\left(96 \nu_{0}^{5}-120 \nu_{0}^{3}+60 \nu_{0}^{2}+245\right) \nu_{0} \cos (\theta)\right. \\
& \left.+576 \nu_{0}^{6}-880 \nu_{0}^{4}+720 \nu_{0}^{3}-300 \nu_{0}^{2}-900 \nu_{0}-15\right) .
\end{aligned}
$$

${ }_{454}$ These ODEs govern the solution for $z_{1}$, the first-order correction to the filament shape. 